\title{
An information theoretical approach to task-switching: evidence from cognitive brain potentials in humans
}

\author{
Francisco Barceló ${ }^{1,2, *}$, José A. Periáñez ${ }^{1,2}$ and Erika Nyhus ${ }^{3}$ \\ 1. Department of Psychology, Universitat de les Illes Balears, Palma de Mallorca, Spain \\ 2. Institut Universitari d'Investigació en Ciències de la Salut, Palma de Mallorca, Spain \\ 3. Department of Psychology, University of Colorado, Boulder, CO, USA
}

Edited by: Robert T. Knight, University of California Berkeley, USA

Reviewed by: Kenneth Hugdahl, University of Bergen, Norway

Kimmo A. Alho, University of Helsinki, Finland

\begin{abstract}
This study aimed to clarify the neural substrates of behavioral switch and restart costs in intermittently instructed task-switching paradigms. Event-related potentials (ERPs) were recorded while participants were intermittently cued to switch or repeat their categorization rule (Switch task), or else they performed two perceptually identical control conditions (NoGo and Oddball). The three tasks involved different task-sets with distinct stimulus-response associations in each, but identical visual stimulation, consisting of frequent colored shapes ( $p=0.9$ ) and randomly interspersed infrequent black shapes ( $p=0.1$; ' + ' and ' $\mathrm{X}$ ' symbols). Behavioral restart costs were observed in the first target responses following all black shapes in the Switch and NoGo tasks - but not in the Oddball task - and corresponded with enhanced fronto-centrally distributed early cue-locked P3 activity (peak latency 325-375 ms post-cue onset at the vertex). In turn, behavioral switch costs were associated with larger late cue-locked P3 amplitudes in the Switch task only (peak latency 400-450 ms post-cue onset at mid-parietal sites). Together with our information theoretical estimations, ERP results suggested that restart and switch costs indexed two neural mechanisms related to the preparatory resolution of uncertainty: (1) the intermittent re-activation of task-set information, and (2) the updating of stimulus-response mappings within an active task set, as indexed by early and late cue-locked P3 activations, respectively. In contrast, target-locked P3 activations reflected a functionally distinct mechanism related to the implementation of task-set information. We conclude that task-switching costs consist of both switch-specific and switch-unspecific processes during the preparation and execution stages of task performance.
\end{abstract}

Keywords: cognitive control, information theory, novelty, set shifting, response uncertainty, working memory

\section{INTRODUCTION}

Humans schedule many daily activities in synchrony with environmental cues. Neuroscientists have recently adopted task-cueing paradigms for exploring the neural dynamics subserving the goal-directed control of human cognition (Barcelo, 2003; Braver et al., 2003; Rushworth et al., 2002), as purportedly different from stimulus-driven control (Näätänen, 1990; Posner and Petersen, 1990). Task-switching involves alternating among 2 or more simple tasks following instruction cues associated with pre-learned sets of action rules or task-sets. In a broad sense, a task set consists of 'if...then' rules governing the mapping between sensory inputs and motor outputs for achieving certain goals (Rubinstein et al., 2001; Schneider and Logan, 2006). At the neural level of description, task-set switching demands online maintenance and updating of

*Correspondence: Francisco Barceló, Department of Psychology, Universitat de les Illes Balears; Institut Universitari d'Investigació en Ciències de la Salut (IUNICS), Ctra. Valldemossa km 7.5, 07122 Palma de Mallorca, Spain. e-mail: f.barcelo@uib.es

Abbreviations: EEG, electroencephalography; ERPs, event-related potentials; SD, standard deviation; SEM, Standard error of the mean; SOA, stimulus onset asynchrony; S-R, stimulus-response; RT, reaction time.

Received: 15 0ctober 2007; paper pending published: 29 November 2007; accepted: 03 January 2008; published online: 28 March 2008

Citation: Front. Hum. Neurosci. (2008) 1: 13. doi: 10.3389/neuro.09.013.2007

Copyright (؟) 2008 Barceló, Periáñez and Nyhus. This is an open-access article subject to an exclusive license agreement between the authors and the Frontiers Research Foundation, which permits unrestricted use, distribution, and reproduction in any medium, provided the original authors and source are credited. sensorimotor associations between stated sets of sensory and motor representations that are to be intermittently updated - either upheld, reversed, or replaced altogether - in a context-sensitive way. Usually subjects respond slower and less accurately when switching than when repeating a task, a phenomenon known as behavioral switch costs (Meiran, 1996; Monsell, 2003).

Most authors now acknowledge a multiplicity of sources for behavioral switch costs. There has been controversy, however, in defining the antecedent conditions and relative contribution from endogenous and switch-specific mechanisms (e.g., endogenous reconfiguration of task set information; cf. Monsell, 2003; Rubinstein et al., 2001), as opposed to exogenous and switch-unspecific mechanisms (e.g., sensory priming and task-set inertia; cf. Allport and Wylie, 2000; Logan and Bundesen, 2003; Schneider and Logan, 2005) to the overall behavioral costs. For instance, switch-unspecific restart costs (also referred to as the 'firsttrial effect'; Allport and Wylie, 2000; Altmann, 2002) are observed when a sequence of trials is unpredictably interrupted with an instructional cue. In such conditions, substantial restart costs to the first target trial following the interrupting signal are typically observed regardless of whether the cue prompts for a switch or a repetition of the previous task. The observation of larger restart costs following task-switches compared to task-repetitions has also been taken as proof of genuine and specific switch costs (Monsell, 2003; Rushworth et al., 2002). Alternatively, these effects have been attributed to a common and switch-unspecific 'strengthening' of cuerelated sensory memories that would be differentially recruited in switch and repeat trials (Altmann, 2002). This type of rationale contends against 
the existence of a dedicated mechanism of endogenous control to account for behavioral switch costs (Logan and Bundesen, 2003; Logan et al., 2007; Schneider and Logan, 2005). In sum, there is controversy as to whether behavioral switch costs owe to unspecific or specific control operations, and to what extent these reflect one or several distinct mechanisms, both at the neural and cognitive levels of explanation.

More recent task-switching and sequence-learning studies support distinct sources of behavioral costs, owing to distinct levels in a putative hierarchy of control. For instance, Lien and Ruthruff (2004) inferred such hierarchical control from the observation that switching between 2 blocks of trials within the same task resulted in larger behavioral costs than switching between 2 different tasks, even though the transition from the first to the second block of trials involved a task repetition (i.e., $X Y-Y X$; where $X$ and $Y$ denote trials from 2 different tasks and the hyphen denotes a temporal gap between trials). The slowing in motor responses observed during the initiation of a sequence of actions is reminiscent of the restart costs observed in intermittently-instructed task-cueing paradigms (Lien and Ruthruff, 2004). These analogies led Schneider and Logan (2006) to consider 2 different levels in a hierarchy of cognitive control, namely (1) the control for initiating an action plan (i.e., a sequence of motor actions); and (2) the control for switching between individual tasks.

A hierarchical architecture of control has been proposed also at the neural level of explanation. Functional brain imaging studies have revealed neural activations during block transitions, as well as when starting and interrupting sequences of trials, that have been explained as endogenous control implemented by lateral prefrontal cortex and/or interconnected brain structures (Fox et al., 2005; Konishi et al., 2001). Koechlin and collaborators (2003) proposed a functional hierarchy of control implemented within the lateral prefrontal cortex. These authors defined 3 levels of control - sensorimotor, contextual, and episodic control - involving a hierarchy of intervening sensorimotor representations linking sensory signals with motor actions (see Koechlin and Summerfield, 2007, for a review). Koechlin and collaborators capitalized on the concept of mutual information between stimuli and responses (or 'input-output covariances'), as had been used in the past for defining the limits of the human capacity for processing information (Miller, 1956). One premise of this approach is that the information tradeoff between sensory and motor representations along a putative hierarchy of cognitive control may be formally estimated using the analytical tools of information theory (Koechlin and Summerfield, 2007; Miller, 1956).

The fast dynamics of sensory-motor interactions during task-switching can be examined with the excellent temporal resolution of event-related potentials (ERPs). A rapidly growing body of evidence lends support to the relationship between task-set switching operations and several cognitive ERPs characterized by their long-latency and positive polarity (e.g., the 'P3' or 'P300' potential; Barcelo, 2003; Barcelo et al., 2006; Karayanidis et al., 2003; Kieffaber and Hetrick, 2005; Kopp et al., 2006; Nicholson et al., 2005; Rushworth et al., 2002). Using a task-cueing paradigm inspired by the Wisconsin card sorting test, Barcelo and collaborators (2002) found that task switch cues elicited robust fronto-parietally distributed 'task-novelty P3' activity whose amplitude, latency, and scalp topography resembled classic 'novelty P3' responses to novel distracters (cf. Friedman et al., 2001). The early, frontal aspect of this cue-locked P3 complex (peak latency $385 \mathrm{~ms}$ at $\mathrm{Fz}$ ) seemed to work in concert with its late, parietally distributed aspect (peak latency $575 \mathrm{~ms}$ at Pz) during the updating of task set information in preparation for the next target response (Barcelo et al., 2002, 2006). Here these 2 aspects of cue-locked P3 activity will be labeled considering their peak latencies ('early' vs. 'late') rather than their scalp distribution ('frontal' vs. 'posterior') (cf. Friedman et al., 2001). Importantly, these cue-locked P3 activations were inversely associated with behavioral efficiency, with larger switch cue-locked P3 activations accompanying larger behavioral costs. In contrast, the $1^{\text {st }}$ target following a task-switch elicited reduced targetlocked P3 amplitudes that increased gradually thereafter accompanying gradual improvements in card sorting efficiency along several repetition trials (Barcelo et al., 2000, 2002). These observations suggested that cuelocked P3 - but not target P3 - potentials indexed transient activation of a fronto-posterior network involved in the preparatory resolution of response uncertainty (cf. Sutton et al., 1965), since cue-locked P3 amplitudes were a function of the amount of task-set information being updated in working memory (Barcelo et al., 2002, 2006). However, these studies could not decide whether cue-locked P3 activations - and the accompanying behavioral costs - indexed switch-specific reconfiguration of appropriate S-R mappings (Rushworth et al., 2002), perhaps related to the stopping and shifting of task-sets (Robbins, 2007), or to a more general switch-unspecific arousal mechanism related to response preparation (Jennings and van der Molen, 2005; Kahneman, 1973), or to the improbable onset of task cues (Donchin, 1981).

The present study aimed to clarify the nature of restart and switch costs in task-switching paradigms (as distinct from other residual 'mixing costs'; Monsell, 2003), and to explore their putative association with concurrent cognitive brain potentials as suggested by recent ERP findings (Barcelo et al., 2002, 2006; Rushworth et al., 2002). In so doing, we formally estimated the mutual information between stimuli and responses to interpret behavioral costs and the accompanying P300 activations, as these had been intuitively associated with uncertainty resolution in the past (Sutton et al., 1965, 1967). From an information theoretical perspective, contextually informative signals in task-cueing paradigms can be assumed to convey information (and demand cognitive control) at least at 2 hierarchically distinct levels of representation, as indexed by restart and switch costs, respectively: (1) lower-order control for dealing with unpredictable task-set information in working memory, as when initiating a new sequence of trials; and (2) higher-order control for updating stimulus-response (S-R) mappings within a currently active task-set. The first type of control relates to the resolution of uncertainty generated by the infrequent onset of any cueing signals initiating/ interrupting a new/old action plan (i.e., both 'switch' and 'repeat' cues; see Figure 1). The second type of control relates to the resolution of uncertainty specifically associated with 'switch' cues for updating appropriate

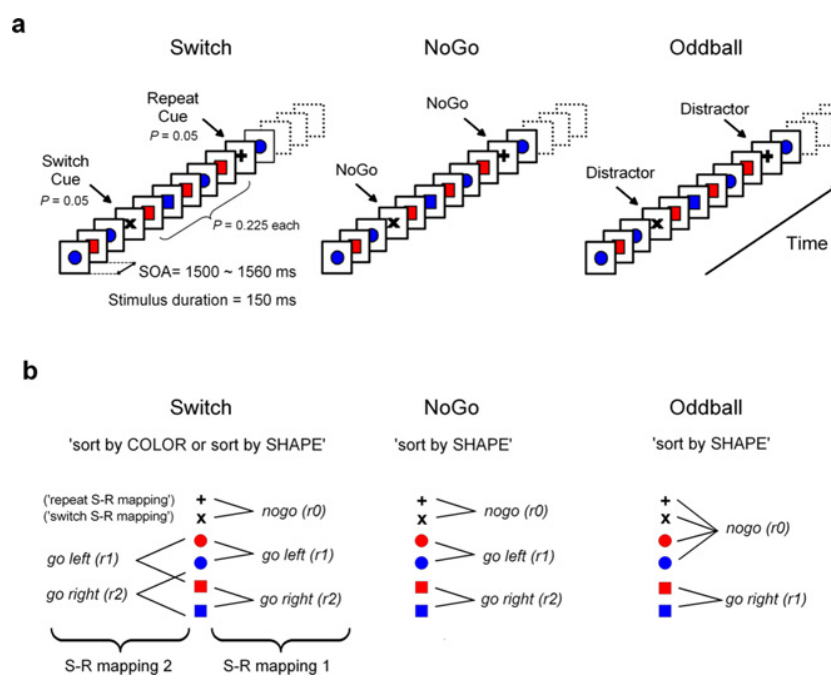

Figure 1. Experimental design and task-set information. (A) Stimulus material. All 3 tasks consisted of the same sequence of frequent colored shapes $(p=0.9)$ with semi-randomly interspersed infrequent black symbols ( $p=0.05$ for both ' + ' and ' $\mathrm{x}$ ' symbols). In the Switch task, symbols ' $\mathrm{x}$ ' and ' + ' instructed subjects to switch and repeat the previous $S-R$ mapping, respectively. The NoGo task consisted of two-forced response choices (i.e., 'press button 1 for circles and button 2 for squares'), whereas the Oddball task involved one-forced response trials (i.e., 'press button 1 for squares'). Subjects were explicitly instructed not to respond to the black symbols ' $\mathrm{x}$ ' and ' + ' in the NoGo and Oddball tasks. (B) Hypothetical task-set information and S-R mappings. Task demands were manipulated (1) by varying the amount of task-set information to be handled in working memory (Oddball vs. NoGo tasks); and (2) by varying the contextual meaning of black symbols for updating the active $S-R$ mappings (NoGo vs. Switch tasks; see Tables 1 and 2). 
S-R mappings in working memory (Barcelo et al., 2006; Koechlin et al., 2003). We employed an intermittently-instructed task-cueing paradigm (Switch task) and 2 perceptually identical control tasks (NoGo and Oddball) to examine behavior and brain activations under 3 identical stimulus contexts, while manipulating response demands at 2 different levels within the putative hierarchy of cognitive control (Barcelo and Knight, 2007; Koechlin and Summerfield, 2007). This design allowed us to dissociate the lowerlevel re-activation of infrequent task-set information from the higher-level updating of appropriate S-R associations. In so doing, the NoGo task comprised an equivalent amount of task-set information as the Switch task, but no explicit demands for updating the active S-R mappings. In turn, the Oddball task consisted of similar sensory information as the other 2 tasks, but with lesser response demands (see Figure 1).

First, we hypothesized that if restart costs reflect the intermittent updating of task-set information in preparation for the next trial sequence, then similar restart costs should be observed in NoGo and Switch tasks, given their equivalent amounts of task-set information (with 6:3 S-R mappings each; see Figures 1 and 2). Conversely, less control and comparatively smaller restart costs would be expected for the 0ddball task with a lesser amount of task-set information (with 6:2 S-R mappings; see Figures 1 and 2 and the "Appendix" section for our estimations of mutual information, stimulus and response entropy). Second, we predicted specific switch costs following switch cues in the Switch task only. Likewise, we predicted that restart and switch costs would correlate with enhancements of early and late cue-locked P3 activations, respectively, as these have been linked to the preparatory resolution of response uncertainty in previous ERP studies (Barcelo et al., 2002; Rushworth et al., 2002). In contrast, response uncertainty was expected to reduce target-locked P3 amplitudes during the implementation of task-set information (task execution). Alternatively, if behavioral switch and restart costs and the

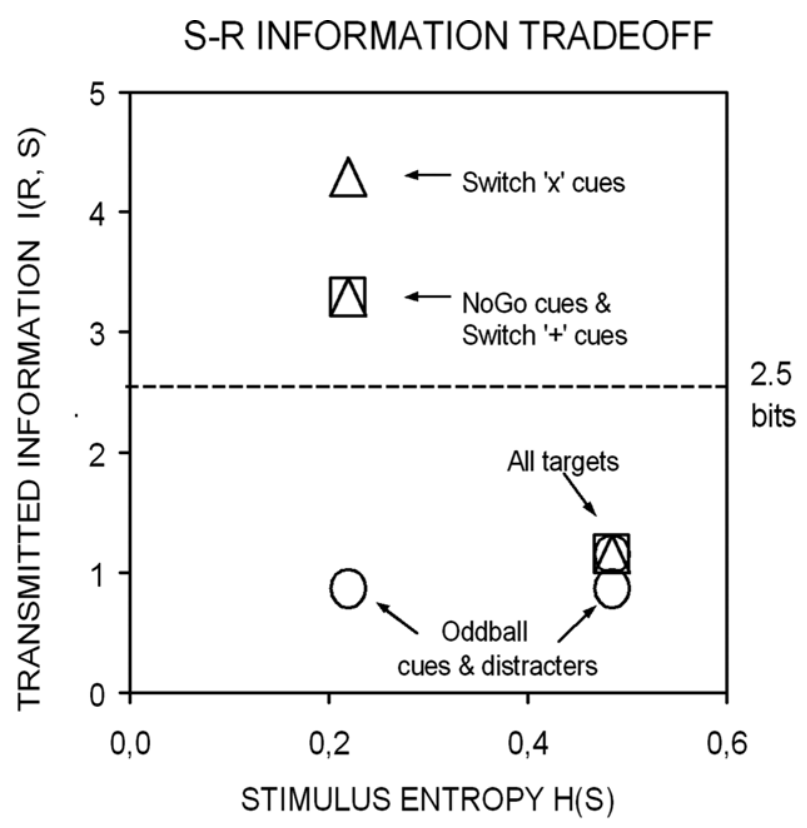

Oddball task

NoGo task

$\triangle$ Switch task

Figure 2. A priori estimations of transmitted information between stimuli and responses as a function of the sensory entropy of black symbols ( 0.22 bits) and colored-shaped stimuli ( 0.48 bits) in the 3 task conditions (after Miller, 1956). Visual targets conveyed the same information for response selection in all 3 task conditions. In turn, black symbols conveyed varying amounts of information for response selection in the Oddball, NoGo and Switch tasks. In the Switch task, ' $x$ ' switch cues conveyed 1 more bit of information than ' + ' repeat cues for updating the active $S-R$ mapping (see Tables 1 and 2 and the "Appendix" section; cf. Koechlin et al., 2003). accompanying P3 activations were primarily determined by sensory encoding and/or sensory priming mechanisms (Altmann, 2002; Logan and Bundesen, 2003), or by purely sensory factors such as the stimulus context (Polich, 2007; Sutton et al., 1967), then we would expect equivalent behavioral costs and transient P3 activations across all perceptually similar tasks.

These predictions were made based on the auditory brain responses to tonal switch and repeat cues from our previous task-cueing studies. Here we extended predictions to the visual modality on the assumption that cognitive P300 potentials index updating of sensorimotor representations in widely distributed multimodal cortical-subcortical networks (Nieuwenhuis et al., 2005; Ranganath and Rainier, 2003; Soltani and Knight, 2000; Sutton et al., 1965). Our information theoretical approach allowed us to address the same task-switching processes employing simpler task procedures and stimulus materials than those in our previous studies (Barcelo et al., 2000, 2002, 2006). This facilitated the estimation of mutual information between stimuli and responses across our different task conditions (Figures 1 and 2, and Tables 1 and 2).

\section{MATERIALS AND METHODS Subjects}

Fifty undergraduate students (32 females, 18 males; mean $\pm S D$ age: $20.7 \pm 3.1$ years) with more than 10 years of education (mean \pm SD: $13.6 \pm 2.5$ years of education) took part in the study. They all had normal or corrected to normal visual acuity and no history of neurological or psychiatric disorders. Subjects were informed about the purpose of the investigation before the experimental session and signed a consent form according to the Declaration of Helsinki. Experimental procedures were in accordance with the Bioethics Committee of the University of the Balearic Islands.

\section{Behavioral procedures}

Participants sat in a comfortable chair in a dimly lit and electrically shielded chamber facing a computer monitor at a distance of 1 minute. Stimuli consisted of 4 equiprobable colored shapes (e.g., red and blue colored circles and squares; $p=0.225$ each) and 2 infrequent black shapes (e.g., '+' and ' $x$ ' symbols; $p=0.05$ each; see Figure 1A). All stimuli had the same spatial dimensions with $6.5 \times 6.5 \mathrm{~cm}$, or $3.8^{\circ} \times 3.8^{\circ}$ of visual angle. Stimulus duration was $150 \mathrm{~ms}$ and stimulus onset asynchrony (SOA) varied randomly between 1500-1560 ms (Figure 1). A single block of trials was constructed and employed in the 3 task conditions. A trial sequence was defined as those colored shapes presented between 2 consecutive black symbols in any of the 3 task conditions. Task stimuli were delivered semi-randomly with the constraint that trial sequences consisted of a varying number of 4 to 8 colored shapes presented between 2 consecutive black symbols. The speed and accuracy of responses were both emphasized in the 3 task conditions. Before the experimental session, subjects performed 1 practice session of 390 trials to ensure they had understood the instructions and reached an asymptote in their learning curve. In the experimental session each subject performed a total of 780 trials distributed in 2 runs of approximately 12 minutes duration each. Upon arrival to the laboratory, subjects were randomly assigned to 1 of the 3 task conditions (Switch, NoGo, Oddball). Therefore, all subjects received the same sequence of visual stimuli, but only 1 of the 3 sets of task instructions. A group design was preferred to a within-subject design in order to avoid any carry-over effects between task conditions (i.e., due to overlearning, to interference between successive task-sets, habituation of transient $\mathrm{P} 3$ responses, etc.).

The Switch task consisted of a variant of the intermittent-instruction paradigm (Monsell, 2003; Rushworth et al., 2002). Seventeen subjects were instructed to sort colored-shapes according to 2 classification rules, either color or shape, by pressing the left and right buttons of a response panel with their left and right index fingers, respectively. When sorting by the color rule, subjects pressed the left button for 'red' 
and the right button for 'blue'. When sorting by the shape rule, subjects pressed the left button for 'circle' and the right button for 'square'. The black symbols ' + ' or ' $x$ ' instructed subjects to either 'repeat' or 'switch' the previous rule, respectively. These simple $S-R$ mappings sufficed for an accurate scoring of behavioral responses within a series of repetition trials, but could result in inaccurate scoring of task-switching errors at rule transition points (i.e., after a black symbol; Barcelo et al., 2000). In order to avoid this, the first and last shapes in a trial sequence always consisted of either a red square or a blue circle. In order to keep perceptual priming effects constant, we controlled for the sequential probabilities between every pair of stimuli. The overall probability of 2 successive switch cues $(\times \ldots \times \ldots)$ was the same as that of 2 successive repeat cues $(+\ldots+\ldots)$. Likewise, the probability of alternations between switch-repeat cues $(\times \ldots+\ldots)$ was the same as the probability of repeat-switch alternations $(+\ldots \times \ldots)$. Finally, black symbols ' $x$ ' and ' + ' instructed the same number of switches and repetitions to the color and shape rules, respectively.

The NoGo task served as a control for the Switch task, presenting similar stimulus and response demands but without the need to reconfigure the stimulus-response mappings (see Figures 1 and 2, Tables 1 and 2, and the "Appendix" section). Sixteen subjects were instructed to sort the same sequence of colored-shapes according to either the color rule or the shape rule in each of 2 experimental runs (e.g., using the color rule in the first run and then the shape rule in the second run, or vice versa). The S-R mappings for the color and shape rules were the same as in the Switch condition (Figure 1B). Additionally, subjects were told to withhold their response to the black symbols. The order of administration of the color and shape runs was counterbalanced across subjects.

The Oddball task was intended to provide a control with an equivalent stimulus context and identical perceptual demands as the Switch and NoGo conditions, but with lesser response demands (see Figures 1 and 2 , Tables 1 and 2, and the "Appendix" section for estimations of the information content of black symbols in each task). Seventeen participants were asked to press a button with their right index finger to any 'square' stimulus in 1 experimental run, and to any 'blue' stimulus in another run. They were also asked to withhold their responses to any other non-target stimulus. The order of administration of the 2 experimental runs was counterbalanced across subjects.

The stimulus material in our tasks was chosen to keep working memory demands and novelty effects to a minimum. In particular, the 6 perceptual items employed in our 3 tasks could be easily memorized and discriminated from each other, and they were selected to meet with the theoretical limits of the human capacity for selecting and holding information in working memory (Miller, 1956).

\section{ERP recordings}

The electroencephalogram (EEG) was recorded from 28 tin electrodes positioned at Fp1, Fp2, Fz, F3, F4, F7, F8, FCz, FC3, FC4, Cz, C3, C4, CP3, CP4, Pz, P3, P4, TP7, TP8, FT7, FT8, T3, T4, T5, T6, 01, and 02, and referenced to the left mastoid. The EEG signal was amplified (band pass, $0.01-30 \mathrm{~Hz} ; 12 \mathrm{~dB} /$ octave roll-off), digitized at $500 \mathrm{~Hz} / \mathrm{channel}$ and stored for off-line averaging. Electrode impedances were kept below $5 \mathrm{k} \Omega$. The vertical and horizontal electrooculogram were also recorded for eye blink correction. Trials with a raw EEG signal outside the range of $\pm 75 \mu \mathrm{V}$ in amplitude, with muscle or any other artifacts in the recording epoch were rejected. Any sequences of trials containing incorrect stimulus classifications, false alarms, and omissions were also discarded from the ERP analyses in all 3 task conditions.

\section{Behavioral analyses}

At the beginning of the Switch task, subjects were instructed to begin with the correct sorting rule up to the onset of the first black symbol in the trial sequence. These trials were eliminated from the analyses. Reaction times (RTs) were measured and analyzed from correct trial sequences only. Any trial sequences containing false alarms, omissions, or any other errors were discarded from the analysis of RTs, although error trials were retained to compute accuracy indexes. Only the first 3 target responses following a black symbol were considered for analysis, since behavioral costs in intermittently-instructed paradigms typically reach an asymptote

Table 1. Mean stimulus probability, $P(S)$, and a priori estimations of mutual information between stimuli and responses, $I(S, R)$, for the 3 tasks in Figure 1. Shannon's (1948) joint entropy $\boldsymbol{H}(\boldsymbol{x}, \boldsymbol{y})=-\sum_{i, j} \boldsymbol{p}(\boldsymbol{i}, \boldsymbol{j}) \log p(\boldsymbol{i}, j)$, and its related notion of mutual information were used to estimate information transmission between sets of stimuli and responses. Only perceptual 'shape' classifications are considered here as an example (also see the "Appendix" section).

\begin{tabular}{lllll}
\hline & All tasks & & $\begin{array}{l}\text { Oddball task } \\
\text { Mutual information I (S, } \boldsymbol{R})\end{array}$ & $\begin{array}{l}\text { NoGo and switch tasks } \\
\text { Mutual information I(S, } \boldsymbol{R})\end{array}$ \\
\hline Task events & Stimulus set & $\boldsymbol{P ( S )}$ & & $I(s 1, r 0)=3.29$ \\
$\mathbf{+}$ & $s 1$ & 0.050 & $I(s 2, r 0)=0.86$ & $I(s 2, r 0)=3.29$ \\
$\mathbf{*}$ & $s 2$ & 0.050 & $I(s 3, r 0)=0.86$ & $I(s 3, r 1)=1.16$ \\
0 & $s 3$ & 0.225 & $I(s 4, r 0)=0.86$ & $I(s 5, r 1)=1.16$ \\
$\mathbf{0}$ & $s 4$ & 0.225 & $I(s 5, r 1)=1.16$ & $I(s 6, r 2)=1.16$ \\
\hline
\end{tabular}

Table 2. Mean stimulus probability, $P(S)$, and a priori estimations of mutual information between contextual stimulus information (CIS) and available S-R mappings, I(SR, CIS), for the $\mathbf{3}$ task-sets in depicted in Figure 1. Shannon's (1948) joint entropy $\boldsymbol{H}(\boldsymbol{x}, \boldsymbol{y})=-\sum_{i, j} \boldsymbol{p}(\boldsymbol{i}, \boldsymbol{j}) \log \boldsymbol{p}(\boldsymbol{i}, \boldsymbol{j})$, and its related notion of mutual information were used to estimate information transmission between black symbols and $S-R$ mappings (cf. "contextual control" in Koechlin et al., 2003).

\begin{tabular}{|c|c|c|c|c|}
\hline \multicolumn{3}{|c|}{ All tasks } & \multirow{2}{*}{$\begin{array}{l}\text { Oddball and NoGo tasks } \\
\text { Mutual information I (SR, C|S) }\end{array}$} & \multirow{2}{*}{$\begin{array}{l}\text { Switch tasks } \\
\text { Mutual information I(SR, C|S) }\end{array}$} \\
\hline Task events & Stimulus set & $P(S)$ & & \\
\hline+ & $s 1$ & 0.050 & $I(s r, \mathrm{c} \mid \mathrm{s} 1)=0.0$ & $I(s r, \mathrm{c} \mid \mathrm{s} 1)=0.0$ \\
\hline$*$ & $s 2$ & 0.050 & $I(s r, \mathrm{c} \mid \mathrm{s} 2)=0.0$ & $I(s r, \mathrm{c} \mid \mathrm{s} 2)=1.0$ \\
\hline
\end{tabular}

4 
Switch

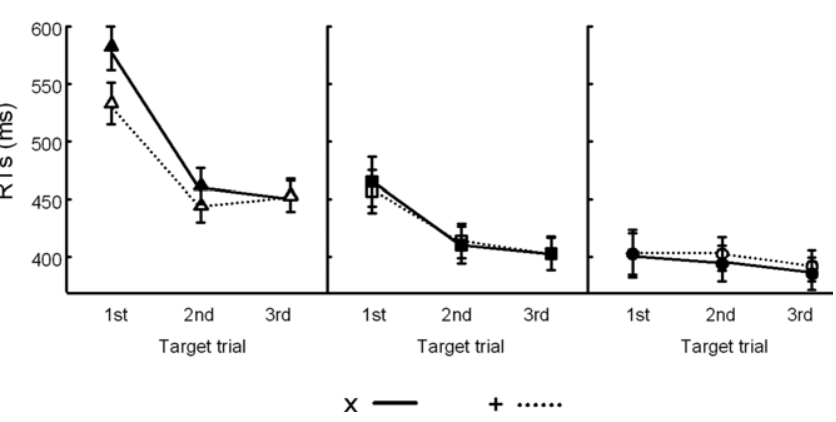

Figure 3. Behavioral results. Mean reaction times (RTS) and SEM to visual targets as a function of their sequential position following black symbols in the 3 task conditions.

in later trials (Monsell, 2003; Rushworth et al., 2002). Following previous studies, we defined 'restart costs' as the mean difference in RTs and errors between the $1^{\text {st }}$ and $2^{\text {nd }}$ target responses following any black symbol. 'Switch costs' were defined as the mean difference in RTs and errors between the $1^{\text {st }}$ target response following an ' $x$ ' cue and the $1^{\text {st }}$ target response following a ' + ' cue.

The percentage of correct target responses and mean RTs from correct target trials were subjected to a 3-way mixed ANOVA, with Black symbol (' $x$ ' vs. ' + ') and Target trial $\left(1^{\text {st }}, 2^{\text {nd }}, 3^{\text {rd }}\right)$ as the within-subjects factors, and Task (Switch, NoGo, Oddball) as the between-subjects factor (see Figure 3).

\section{ERP analyses}

ERP waveforms were computed off-line using a linked-mastoid reference. ERPs in the Switch, NoGo, and Oddball tasks were time-locked to the onset of black symbols (' $x$ ' vs. ' + '), as well as to the $1^{\text {st }}, 2^{\text {nd }}$, and $3^{\text {rd }}$ targets following each black symbol. Epoch length was $650 \mathrm{~ms}$ including a $50 \mathrm{~ms}$ baseline. This short baseline was adopted to minimize the effects of prebaseline shifts in some conditions (Karayanidis et al., 2003). A minimum of 40 clean EEG epochs contributed to individual cue-locked ERP waveforms (' $x$ ' mean $=47.9$ epochs; ' + ' mean $=48$ epochs). A minimum of 65 clean EEG epochs contributed to individual target-locked ERP waveforms (' $x$ ' mean $=115.5$; ' + ' mean $=115.9$, with $1^{\text {st }}, 2^{\text {nd }}$, and $3^{\text {rd }}$ target trials collapsed). Mean P3 amplitudes were computed after visual inspection of the grand averages of cue-locked and target-locked ERP waveforms, and coincided with the peak latency of these P3 sub-components (Figure 4). For the cue-locked ERPs, the mean amplitude of the early aspect of the P3 component was measured in a 50-ms window around its peak latency at $\mathrm{Cz}$ (325-375 ms post-cue onset). The mean amplitude of the late aspect of the $\mathrm{P} 3$ component was measured in a $50-\mathrm{ms}$ window around its peak latency at $\mathrm{Pz}$ (400-450 ms post-cue onset). Mean target-locked $\mathrm{P} 3$ amplitudes were measured in a $50-\mathrm{ms}$ window around their peak latency at $\mathrm{Pz}$ (325-375 ms post-target onset; Figure 4).

Cue-locked P3 potentials were analyzed using a 3-way mixed ANOVA with Task (Switch, NoGo, Oddball) as the between-subject factor, and Black symbol (' $\mathrm{x}$ ' vs. ' + '), and Electrode site ( $\mathrm{Fz}, \mathrm{Cz}, \mathrm{Pz}$ ) as the within-subject factors. Target-locked P3 potentials were analyzed with a similar statistical design (with the brain responses to $1^{\text {st }}, 2^{\text {nd }}$ and $3^{\text {rd }}$ targets collapsed). A mixed ANOVA was used to test differences in the scalp distribution of early and late cue-locked P3 and target-locked P3 activations (Figure 5). The within-subject factors in this ANOVA considered three P3 sub-components (early P3, late P3, target P3), 3 Electrodes $(\mathrm{Fz}, \mathrm{Cz}, \mathrm{Pz}$ ), plus the between-subject factor Task (NoGo, Oddball and Switch tasks). Sequential trial effects were examined with a mixed ANOVA performed on consecutive $1^{\text {st }}, 2^{\text {nd }}$ and $3^{\text {rd }}$ target trials following

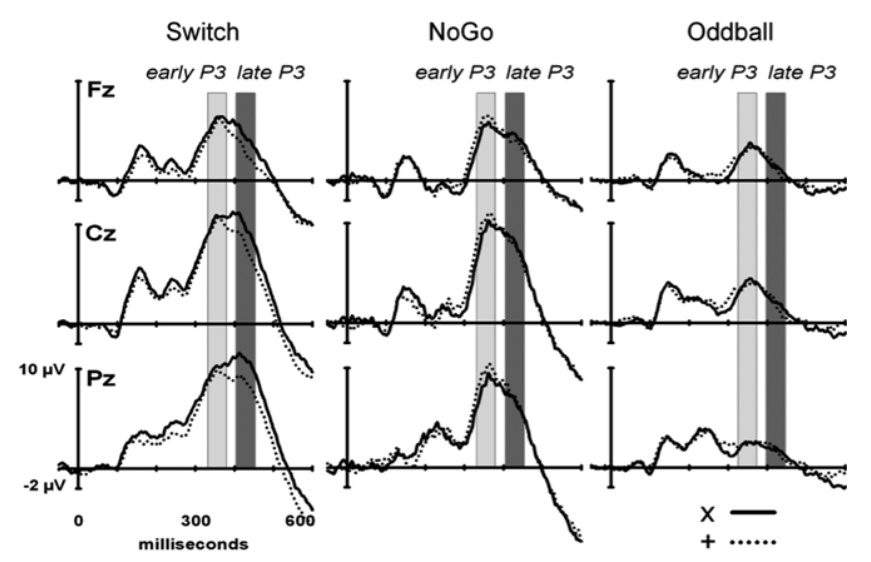

b

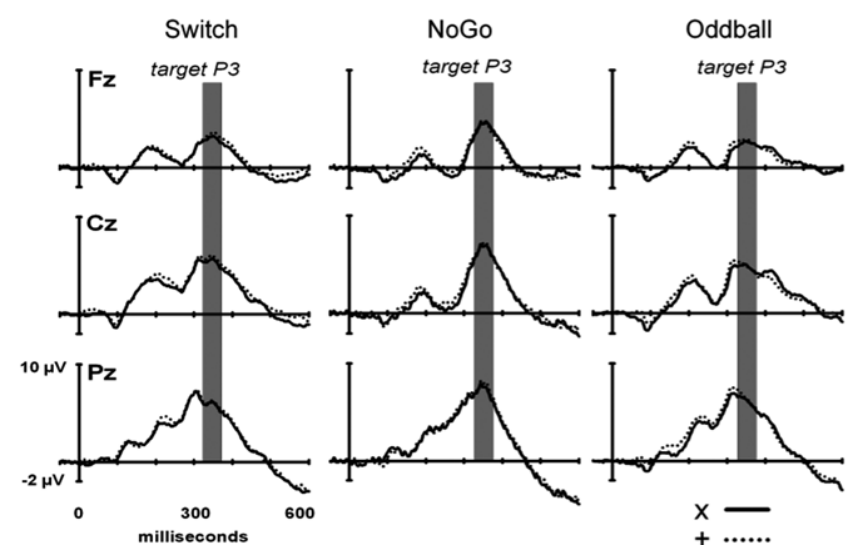

Figure 4. Event-related potentials (ERPs) at 3 midline electrodes ( $\mathrm{Fz}, \mathrm{Cz}$, Pz). (A) Grand cue-locked ERPs elicited by black symbols ' $\mathrm{X}$ ' and ' + ' in the Switch, NoGo, and Oddball conditions. (B) Grand target-locked ERPs to black symbols ' $\mathrm{x}$ ' and '+' in the Switch, NoGo, and Oddball task conditions (with 1st, 2nd and 3rd targets trials collapsed). Gray bars show the latency windows for amplitude measurements of the early and late aspects of cue-locked P3 activity, and for target-locked P3 activity.

black symbols in the Switch and NoGo tasks with the between-subject factor Task (Switch vs. NoGo), and 3 within-subject factors: Black symbol (' $x$ ' vs. '+'), Target trial $\left(1^{\text {st }}, 2^{\text {nd }}, 3^{\text {rd }}\right)$, and Electrode $(F z, C z, P z)$.

\section{Regression and correlation analyses}

A series of linear regression analyses were conducted to explore the association between behavioral costs and transient P3-like activations. An index of behavioral restart costs was computed as the difference in RTs between $1^{\text {st }}$ and $2^{\text {nd }}$ target responses following all black symbols according to the formula: $1 / 2 \times\left[\left(1^{\text {st }}\right.\right.$ target after ' $x$ ' $+1^{\text {st }}$ target after ' + ' $)-\left(2^{\text {nd }}\right.$ target after ' $x$ ' $+2^{\text {nd }}$ target after ' + ')]. A behavioral index of specific switch costs was computed as the difference in RTs between the $1^{\text {st }}$ target response following black symbols ' $x$ ' and ' + ' in all task conditions. These regression analyses considered data from $1^{\text {st }}$ and $2^{\text {nd }}$ target trials after any black symbol, allegedly showing the largest behavioral costs and strongest associations with cue-locked and target-locked P3 activations. An association of restart and switch costs with these P3-like potentials would suggest a role of those brain responses in the generation of the corresponding behavioral costs. However, such an association would also be expected based on differences in effort or arousal during task performance (Kahneman, 1973). In order to examine the null hypothesis that transient P3-like activations reflect general effort or arousal rather than 

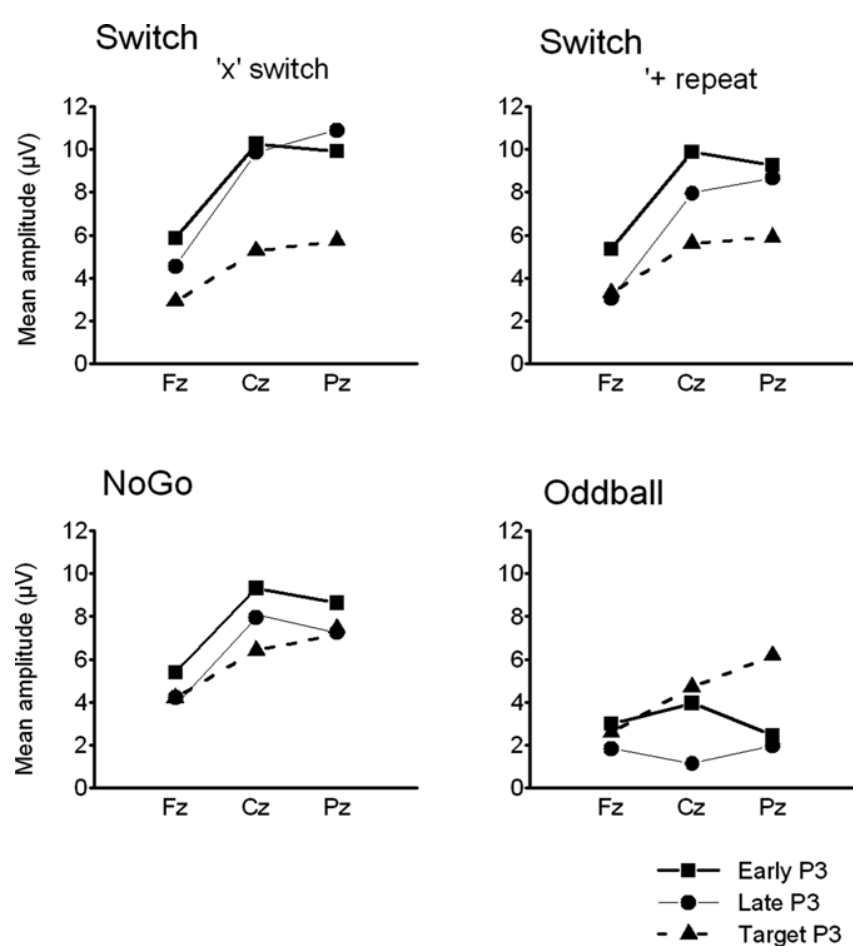

Figure 5. Summary of grand mean amplitudes from 3 midline electrodes (Fz, Cz, Pz) of early and late cue-locked P3 and target-locked P3 activations in the 3 task conditions: Switch (comparing ' $x$ ' switch and ' + ' repeat trials), NoGo and Oddball tasks (with ' $x$ ' and ' + ' trials collapsed). Note the distinct scalp distribution of cue-locked P3 - but not of target-locked P3 - activity across the 3 task conditions.

specific task-switching mechanisms, mean RTs to the $1^{\text {st }}$ target following a black symbol were also considered as a criterion in a third regression model. In testing each regression model (restart costs, switch costs, mean RTs), we searched for the best fit both within each task condition, as well as among those tasks where such an association was observed or predicted (i.e., the null hypothesis of general arousal predicts a negative association between mean RTs and mean P3 amplitudes in all 3 task conditions). Mean cue-locked P3 and target-locked P3 amplitudes measured at 3 midline electrodes ( $\mathrm{Fz}, \mathrm{Cz}, \mathrm{Pz}$ ) were used as predictors. Finally, in order to explore our hypothesis that early and late cue-locked P3 activations reflect an integrated system for updating task-set information, in sharp contrast to target-locked P3 activations (Barcelo et al., 2002), we carried out a series of bivariate Pearson product-moment correlations between our 3 indexes of brain activation. Alternatively, if parietally distributed late cue-locked P3 amplitudes compared to target-locked P3, then these 2 indexes should be more strongly correlated than the early and late aspects of cue-locked P3 (cf. Polich, 2007).

SPSS v14.0 statistical software was used, and a significance level of $p<0.05$ was adopted for all statistical analyses. The level of significance was adjusted when necessary with a Greenhouse-Geyser (G-G) correction against violation of the assumption of homogeneity in the variance of the means. A Bonferroni-corrected significance level of $p<0.05$ was also adopted for all tests of simple effects involving multiple comparisons.

\section{Information theoretical analyses}

We adopted an information theoretical approach to task-switching, whereby we obtained a priori estimations of the mutual information between sensory stimuli and motor responses in our tasks in order to guide our interpretation of behavioral and ERP results (cf. Barcelo and Knight, 2007). In so doing, we followed original recommendations by Miller (1956) for estimating the amount of information transmitted between contextually related stimuli and responses (or input-output correlations) along a putative hierarchy of cognitive control (Koechlin and Summerfield, 2007). These information-theoretic analyses led us to define the informational structure of our task conditions in terms of overall stimulus and response entropies, as well as in terms of the mutual information between contextually related targets, non-targets and motor responses in each of our 3 tasks (Miller, 1956). A summary of these information theoretical estimations is shown in Figure 2 and in Tables 1 and 2. A more detailed report of these estimations can be found in the Appendix Section (cf. Barcelo and Knight, 2007; Koechlin and Summerfield, 2007).

\section{RESULTS}

\section{Behavioral results}

Accuracy. The 3 tasks were performed very efficiently, as confirmed by the analysis of correct responses. Subjects scored an average of $94.1 \%$ correct trials in the Switch task, $95.7 \%$ in the NoGo task, and $98.2 \%$ in the Oddball task. A main effect for Task $\left(F_{(2,47)}=19.8, p<0.0001\right)$, revealed a higher percentage of correct responses in the 0ddball task compared to the other 2 tasks $(p<0.001)$, but no differences between the Switch and NoGo tasks. An interaction between Task, Black symbol, and Target trial $\left(F_{(4.94)}=7.1, p<0.0001\right)$ revealed larger error rates in the first target responses following a ' $x$ ' switch cue as compared to a '+' repeat cue in the Switch task ( $4.2 \%$ vs. $3.5 \%$, respectively, $p<0.04)$. Mean error rates did not differ between black symbols ' $x$ ' and ' + ' in later trials, nor in the other 2 control conditions. The interaction Task by Target trial $\left(F_{(2.4)}=18.4, p<0.0001\right)$, revealed larger error rates in the first than the second target responses following any black symbol in both the Switch and NoGo tasks (mean $1^{\text {st }}$ vs. $2^{\text {nd }}$ trial difference: $2.5 \%$ vs. $1.6 \%$, respectively), but not in the Oddball task.

Reaction times (RTs). The analysis of RTs yielded significant main effects for all 3 factors: Task $\left(F_{(2,47)}=9.4, p<0.0001\right)$, Black symbol $\left(F_{(1.47)}=7.9, p<0.007\right)$ and Target trial $\left(F_{(2,94)}=86.6, p<0.0001\right.$, $\mathrm{G}-\mathrm{G}=0.67$; see Figure 3). An interaction between Task and Target trial $\left(F_{(4,94)}=21.6, p<0.0001, \mathrm{G}-\mathrm{G}=0.67\right)$ revealed the presence of restart costs in the Switch and NoGo tasks, but not in the Oddball task. In both the Switch and NoGo tasks, subjects responded slower to the $1^{\text {st }}$ than $2^{\text {nd }}$ or $3^{\text {rd }}$ target trials $(P s<0.0001$ for all contrasts), with no differences between $2^{\text {nd }}$ and $3^{\text {rd }}$ target trials. An interaction between Task and Black symbol $\left(F_{(2,4)}=13.1, p<0.0001\right)$ revealed reliable switch costs in the Switch task in response to targets following ' $\mathrm{x}$ ' switch vs. ' + ' repeat cues $(p<0.0001)$, but not in the NoGo $(p=0.6)$ nor Oddball tasks $(p=0.2)$. A 3-way interaction between Task, Black symbol, and Target trial $\left(F_{(4,94)}=4.4, p<0.003, \mathrm{G}-\mathrm{G}=0.95\right)$, revealed a multiplicative association between restart and switch costs, as well as the presence of mixing costs in the Switch task (Monsell, 2003), but not in the 2 control tasks. The multiplicative association between restart and switch costs in the Switch task could be inferred from the larger switch costs to the $1^{\text {st }}$ than to the $2^{\text {nd }}$ targets (mean switch cost to $1^{\text {st }} v s$. $2^{\text {nd }}$ target trials $=49.5$ vs. $17.0 \mathrm{~ms}, p<0.008$ ). Mixing costs were inferred from the larger mean RTs to $3^{\text {rd }}$ target trials in the Switch task as compared to the control tasks $(P s<0.04$; Figure 3$)$.

\section{ERP results}

Cue-locked P3 (early aspect, early P3). A main effect of Task $\left(F_{(2,4)}=11.9, p<0.0001\right)$ revealed larger amplitudes of early P3 activity in the Switch and NoGo tasks as compared to the Oddball task ( $P s<0.001)$, with no significant differences in early P3 amplitudes between Switch and NoGo tasks. Neither the Black symbol factor $\left(F_{(1,45)}<1\right)$, nor its interaction with other factors modulated early P3 activity (see Figure 4A). An interaction between Task and Electrode $\left(F_{(4.90)}=8.6, p<0.0001, \mathrm{G}-\mathrm{G}=0.77\right)$ revealed different scalp distributions of early P3 activity for different tasks. Early P3 amplitudes showed a maximum at the vertex (Cz) in all 3 task 
conditions (Figures 4 and 5). However, the comparatively reduced early P3 amplitudes over parietal regions in the Oddball task $(P s<0.0001)$ were responsible for a relatively fronto-central scalp distribution of early P3 amplitudes in this task as compared with their more central-parietal scalp distribution in the Switch and NoGo tasks (Figures 4A, 5, 7).

Cue-locked P3 (late aspect, late P3). The main effects for Task and Black symbol reached significance $\left(F_{(2,4)}=18.9, p<0.0001\right.$, and $F_{(1,45)}=8.6, p<0.005$, respectively; see Figure 4A). An interaction between Task and Black symbol $\left(F_{(2,45)}=4.7, p<0.014\right)$ revealed larger late P3 amplitudes in response to switch as compared to repeat cues in the Switch task only $(p<0.0001)$. In turn, late P3 responses to ' $\mathrm{X}$ ' and ' + ' black symbols did not differ in amplitude in the NoGo $(p=0.8)$ nor the Oddball tasks $(p=0.5)$. The analysis of simple effects of the interaction Task by Electrode $\left(F_{(4.90)}=10.9, p<0.0001, \mathrm{G}-\mathrm{G}=0.65\right)$, revealed that late $\mathrm{P} 3$ amplitudes peaked at central-parietal sites in both the Switch and NoGo tasks, whereas no such differences were apparent across midline electrodes in the 0ddball task ( $P s>0.5)$. The interaction between Task, Black symbol and Electrode did not reach significance (Figures 4A, 5 and 7).

Target-locked P3. A main effect for Electrode revealed significant differences in target-locked P3 activity across the 3 midline electrodes $\left(F_{(2,90)}=65.6, p<0.0001, \mathrm{G}-\mathrm{G}=0.79\right)$, with maximal target $\mathrm{P} 3$ amplitudes recorded at $\mathrm{Pz}(P s<0.005$, for all pairwise contrasts among electrodes; see Figures 4B, 5 and 7 ). The association between behavioral restart costs and the modulations of target-locked P3 activity over successive Target trials was further explored with a mixed ANOVA with the group factor Task (Switch, NoGo), and within-subjects factors Black symbol (' $X$ ' vs. ' + '), Target trial $\left(1^{\text {st }}, 2^{\text {nd }}, 3^{\text {rd }}\right)$ and Electrode (Fz, Cz, Pz). Main effects for Target trial $\left(F_{(260)}=6.4, p<0.003, \mathrm{G}-\mathrm{G}=0.89\right)$ and Electrode $\left(F_{(2,60)}=35.7, p<0.0001, \mathrm{G}-\mathrm{G}=0.72\right)$, as well as the interaction Target trial by Electrode $\left(F_{(4.120)}=4.3, p<0.016, \mathrm{G}-\mathrm{G}=0.53\right)$, revealed an increase in target-locked P3 amplitude from $1^{\text {st }}$ to $2^{\text {nd }}$ target trials $(p<0.0001$ at $\mathrm{Cz}$; Figures 6 and 7$)$. There was no difference in targetlocked P3 amplitudes between $2^{\text {nd }}$ and $3^{\text {rd }}$ target trials after a black symbol. No other main effect or interactions reached significance (Figures 6 and 7). The 0ddball task was not considered in this analysis because it did not show restart costs nor sequential target $\mathrm{P} 3$ effects.

Functional segregation of P3-like brain activations. The 3-way interaction between P3 sub-components (early P3, late P3, target P3), Task (Switch, NoGo, Oddball), and Electrode (Fz, Cz, Pz), was significant $\left(F_{(6,130)}=9.3, p<0.0001, \mathrm{G}-\mathrm{G}=0.72\right)$, suggesting that these P3 subcomponents were differently influenced by task conditions and site of recording (Figure 5). Early cue-locked P3 amplitudes revealed main effects for Task $\left(F_{(2,45)}=12.7 ; p<0.0001\right)$, and Electrode $\left(F_{(2,90)}=40.7\right.$, $p<0.0001, \mathrm{G}-\mathrm{G}=0.75)$, as well as for their interaction $\left(F_{(4.90)}=8.7\right.$, $p<0.0001$ ), indicating differential early cue-locked P3 activations between the 0ddball task and the other 2 tasks at all mid-line electrodes $(P s<0.02)$, but no differences in the scalp distribution of early cue-locked P3 activations were found between the Switch and NoGo tasks ( $P s>0.4$; Figures $4 \mathrm{~A}$ and 5 ). Late cue-locked P3 amplitudes showed main effects for Task $\left(F_{(2,45)}=21.3, p<0.0001\right)$, Electrode $\left(F_{(2,90)}=53.4, p<0.0001\right.$, $\mathrm{G}-\mathrm{G}=0.65)$, and their interaction $\left(F_{(4,90)}=11.9, p<0.0001\right)$, suggesting a different distribution of neural generators across task conditions. Late cue-locked P3 amplitudes were larger in the NoGo task as compared to the Oddball task at all midline sites $(P s<0.0001$; Figures $4 \mathrm{~A}$ and 5), but differences between Switch and NoGo tasks were apparent at the mid-parietal region only ( $p<0.02$; with $10.9 \mu \mathrm{V}$ and $6.9 \mu \mathrm{V}$ in the Switch and NoGo tasks, respectively). In contrast, target-locked P3 amplitudes showed a main effect for Electrode $\left(F_{(2,90)}=67.7, p<0.0001\right.$, $\mathrm{G}-\mathrm{G}=0.82$ ), but not for Task nor any interaction with the other factors, suggesting similar scalp distributions of target-locked P3 activity for all 3 task conditions (Figures 4B and 5).

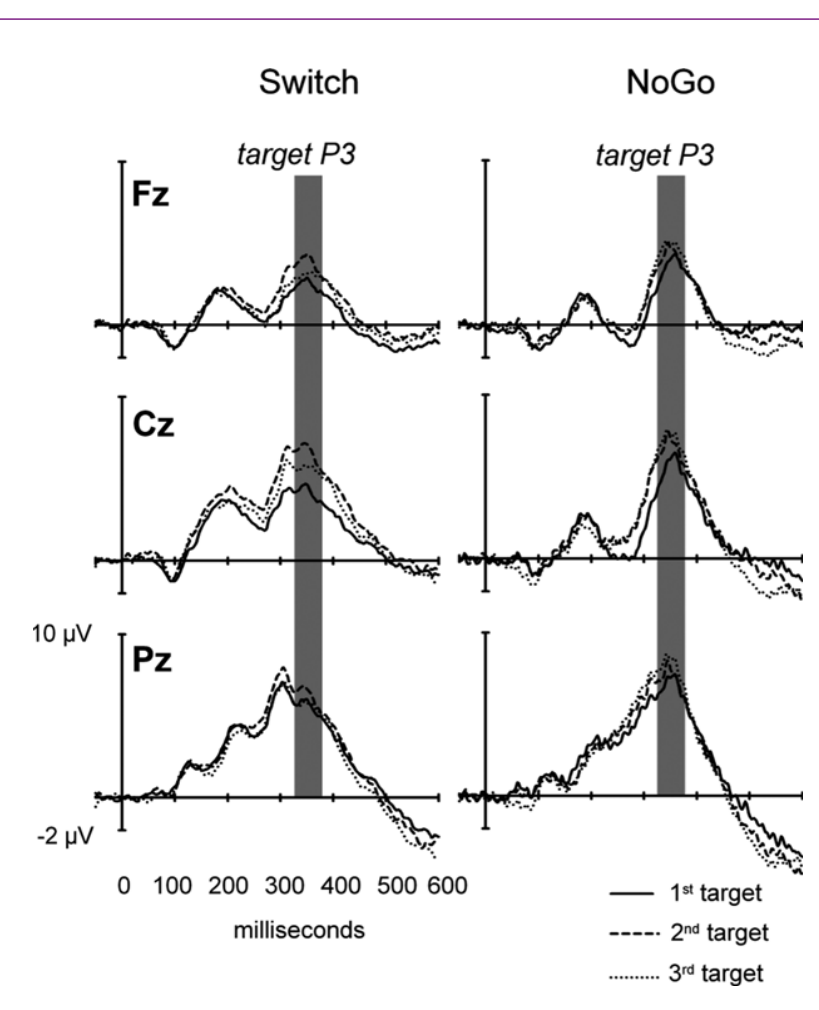

Figure 6. Target-locked ERPs to 3 consecutive targets trials in the Switch and NoGo tasks. Grand ERP waveforms elicited by the $1^{1 s t}, 2^{\text {nd }}$ and $3^{\text {rd }}$ targets in a sequence of trials (with ' $\mathrm{x}$ ' and '+' trials collapsed). The data are shown from 3 midline electrodes ( $F z, C z, P z)$. Gray bars indicate the latency window for measurement of mean target-locked $P 3$ amplitudes.

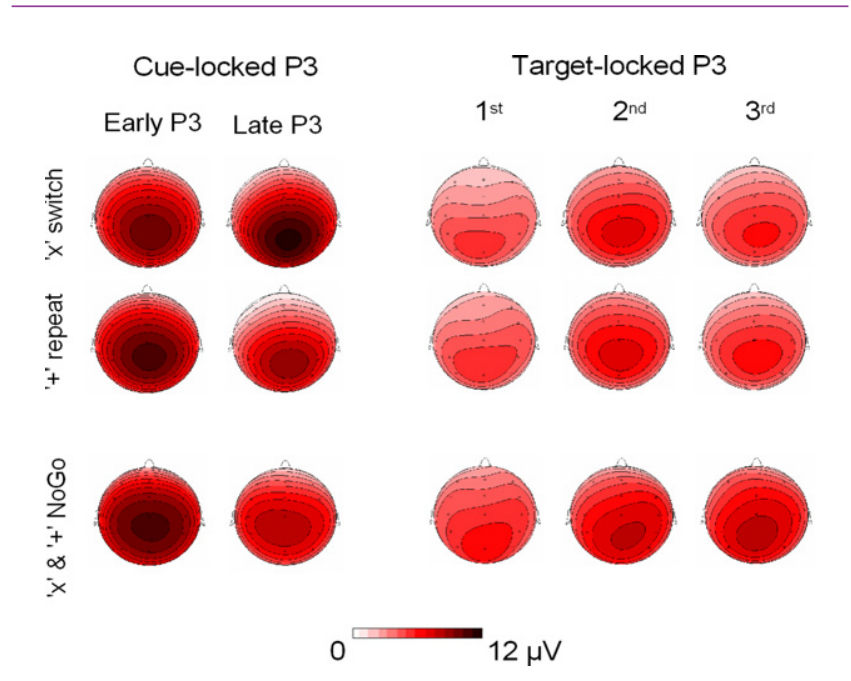

Figure 7. Scalp topography of cue-locked P3 and target-locked P3 amplitudes in the Switch (upper 2 rows) and NoGo tasks (lower row, with ' $x$ ' and ' + ' trials collapsed). The topographic maps for cue-locked $P 3$ activity were computed from the mean voltages of early P3 (325-375 ms) and late $P 3(400-450 \mathrm{~ms})$, respectively. Topographic maps for target $P 3$ activity (325-375 ms) are displayed for the 1st, 2nd, and 3rd target trials following a black symbol.

\section{Association of behavioral costs with transient P3-like activations}

A series of multiple regression analyses examined how much of the variance in behavioral costs and mean RTs was explained by cue-locked P3 and target-locked P3 activations. To minimize the number of predictor variables, we used the mean amplitude of early and late cue-locked P3 and 
target-locked P3 measured at the mid-central electrode (Cz), since these provided the best fits with behavior and were strongly correlated with brain activity from other midline sites. For each of the 3 regression models, the dependent variable was 1 of the 3 behavioral indicators described in the "Materials and methods" section (restart costs, switch costs, mean RTs). In a first stage, we adopted a stepwise regression procedure whereby predictors successively entered the model on the basis of the strength of their association with the dependent variable, after being covaried for the effects of variables already in the model. At a second stage, a forcedentry procedure was adopted to evaluate the strength of the association of predictors with behavior after partialling out any co-linearities. Model estimation ended when no other variable met a fixed criterion for entry $(p=0.10)$. In testing each regression model, we searched for the best fit both within each task condition, as well as across those tasks where an association had been observed or predicted (i.e., model 2 was tested in the Switch and NoGo tasks where restart costs had been observed). Table 3 presents a summary of these regression analyses. Table 4 presents a summary of product-moment correlations between the predictors in our 3 models, suggesting that the correlations between predictors strongly depended on task condition.

Switch-specific behavioral costs were significantly associated with mean late cue-locked P3 'switch costs' computed as the difference in

Table 3. Summary of multiple linear regression analyses. The best fits were obtained for data from the Switch (models 1 and 2) and Nogo tasks (model 2). None of the 3 models fitted data from the Oddball task.

\section{Regression models and predictors}

Overall $R^{2}$ (adjusted $R^{2}$ ), $F$ statistic, degrees of freedom (regression, residual), and partial standardized beta $(\beta)$
Best fit, single task Best fit, across tasks

\begin{tabular}{|c|c|c|}
\hline Model 1: Switch costs & $R^{2}=0.52(0.41), F_{3,13}=4.7 *$ & - \\
\hline Cue-locked early P3 ${ }^{\dagger}$ & $\beta=-0.26 \mathrm{~ns}$ & - \\
\hline Cue-locked late P3 & $\beta=-0.39 * \mathrm{~ns}$ & - \\
\hline $1^{\text {st }}$ target $P 3^{\dagger}$ & $\beta=-0.67 *$ ns & - \\
\hline Model 2: Restart costs & $\boldsymbol{R}^{2}=0.68(0.60), \boldsymbol{F}_{3,12}=8.4 * *$ & $R^{2}=0.27(0.19), F_{3,29}=3.5^{*}$ \\
\hline Cue-locked early P3 & $\beta=1.10 * *$ & $\beta=0.46 *$ \\
\hline Cue-locked late P3 & $\beta=0.02 \mathrm{~ns}$ & $\beta=0.10 \mathrm{~ns}$ \\
\hline $1^{\text {st }}$ target $P 3$ & $\beta=-0.44 \mathrm{~ns}$ & $\beta=-0.69 \mathrm{~ns}$ \\
\hline Model 3: Mean RTs & $R^{2}=0.58(0.48), F_{313}=5.9 *$ & $\boldsymbol{R}^{2}=0.37(0.31), \boldsymbol{F}_{3.29}=5.8^{* * *}$ \\
\hline Cue-locked early P3 & $\beta=-0.17 \mathrm{~ns}$ & $\beta=0.30 \mathrm{~ns}$ \\
\hline Cue-locked late P3 & $\beta=0.04 \mathrm{~ns}$ & $\beta=0.02 \mathrm{~ns}$ \\
\hline $1^{\text {st }}$ target P3 & $\beta=-0.67^{\star \star}$ & $\beta=-0.77 * * *$ \\
\hline
\end{tabular}

(ns, non significant).

$* P<0.05$.

$* * P<0.01$.

$* * * P<0.001$

Switch minus repeat trial differences in P3 amplitude were used instead of mean P3 amplitudes.

Table 4. Pearson product-moment correlations between mean P300 amplitudes measured at Cz.

\begin{tabular}{|c|c|c|c|c|}
\hline & Cue-locked early P3 & Cue-locked late P3 & $1^{\text {st }}$ target P3 & $3^{\text {rd }}$ target P3 \\
\hline \multicolumn{5}{|l|}{ Switch task $(N=17)$} \\
\hline Cue-locked early P3 & 1.00 & $0.75^{* *}$ & $0.66 * *$ & $0.79 * *$ \\
\hline Cue-locked late P3 & - & 1.00 & $0.54 *$ & $0.76 * *$ \\
\hline $1^{\text {st }}$ target $\mathrm{P} 3$ & - & - & 1.00 & $0.73 * *$ \\
\hline \multicolumn{5}{|l|}{ NoGo task $(N=16)$} \\
\hline Cue-locked early P3 & 1.00 & $0.51 *$ & $0.76 * *$ & $0.86 * *$ \\
\hline Cue-locked late P3 & - & 1.00 & $0.59 *$ & $0.78 * *$ \\
\hline $1^{\text {st }}$ target $\mathrm{P} 3$ & - & - & 1.00 & $0.82 * *$ \\
\hline \multicolumn{5}{|l|}{ Oddball task $(N=17)$} \\
\hline Cue-locked early P3 & 1.00 & $0.54 *$ & $0.51 * *$ & $0.35 \mathrm{~ns}$ \\
\hline Cue-locked late P3 & - & 1.00 & $-0.07 \mathrm{~ns}$ & $-0.25 \mathrm{~ns}$ \\
\hline $1^{\text {st }}$ target $\mathrm{P} 3$ & - & - & 1.00 & $0.62 * *$ \\
\hline \multicolumn{5}{|c|}{ Across all tasks $(N=50)$} \\
\hline Cue-locked early P3 & 1.00 & $0.76 * *$ & $0.44 * *$ & $0.67 * *$ \\
\hline Cue-locked late P3 & - & 1.00 & $0.21 \mathrm{~ns}$ & $0.52 * *$ \\
\hline $1^{\text {st }}$ target $\mathrm{P} 3$ & - & - & 1.00 & $0.68 * *$ \\
\hline
\end{tabular}

$$
\begin{aligned}
& * P<0.05 \text {. } \\
& * * P<0.01 \text { (2-tailed). }
\end{aligned}
$$

8 
amplitude of late P3 to switch vs. repeat symbols in the Switch task (Table 3, model 1). However, the strongest predictor of specific switch costs was mean target P3 amplitude, that raised the explained variance from 15 to $52 \%$ compared to the previous predictor. In both cases, larger 'switch minus repeat' differences in late cue-locked P3 and target P3 activations predicted smaller switch costs. Model 1 did not fit data from the NoGo and Oddball tasks (Table 3).

Behavioral restart costs were explained by the mean amplitude of early cue-locked P3 activity in the NoGo and Switch task conditions (Table 3, model 2). Mean early cue-locked P3 amplitudes in the NoGo task provided the best fit to model 2 , with $60 \%$ of explained variance in restart costs, with larger early P3 amplitudes predicting larger restart costs. Inclusion of the other 2 predictors yielded a non-significant $7.8 \%$ increase of explained variance in the model, suggesting that their contribution to restart costs was negligible after partialling out their correlation with early P3 activity. The strength of this association was further reduced (to only $27 \%$ of explained variance) when data from the Switch task entered the model, suggesting that restart costs in the more complex Switch task were tapping into distinct sources of variability as those in the NoGo task.

Model 3 considered mean RTs to the $1^{\text {st }}$ target trial as the criterion, since these were highly correlated with overall RTs $(r=0.96, p<0.01$ for the whole sample of $N=50$ ). The best single task fit for mean RTs was contributed by mean target P3 amplitudes to the $1^{\text {st }}$ target in the Switch task (with $58 \%$ of explained variance), and this did not improve significantly when considering subjects from the other 2 task conditions. In particular, none of the 3 regression models reached significance for data from the Oddball task, suggesting a weak association between transient P3-like activations and behavior in this task condition.

Target-locked P3 activity always showed an inverse relationship with all behavioral indexes, while also being directly associated with early and late cue-locked P3 amplitudes. The relative strength of the association between all 3 brain indexes did not depend on the temporal proximity between the measures, as suggested by the stronger correlations between $1^{\text {st }}$ and $3^{\text {rd }}$ target P3 activations, as compared with early and late cuelocked P3 activations (e.g., NoGo and Oddball tasks, Table 4). These relatively strong correlations among all 3 brain indexes need to be taken into consideration when interpreting results from the regression analyses.

\section{DISCUSSION AND CONCLUSIONS}

In accord with the extant literature on task-switching, we observed behavioral costs consisting of slower and less accurate target responses when participants intermittently switched their classification rule. Importantly, we found response costs in speed and accuracy in the context of task repetitions as well as of task switches. A comparison with 2 perceptually identical control tasks conveying equivalent amounts of stimulus entropy, but involving different response demands (Figures 1 and 2, and Tables 1 and 2), allowed us to fractionate the overall behavioral costs into 2 distinct portions that were also related to distinct transient P3-like activations. First, large switch-unspecific restart costs were measured to the $1^{\text {st }}$ target responses following all black symbols in the Switch and NoGo tasks, and regardless of the explicit meaning of those signals for updating the currently active S-R mappings. Second, a smaller switchspecific switch cost was related to the meaning of ' $x$ ' switch symbols in the Switch task for updating the currently active S-R mapping in working memory. Behavioral restart and switch costs were accompanied by enhanced early and late cue-locked P3 activations, respectively. In contrast, target-locked P3 amplitudes were significantly reduced to the $1^{\text {st }}$ target following all black symbols in the Switch and NoGo tasks, but did not differ between tasks in the $2^{\text {nd }}$ and later repetition trials. These findings support a critical functional dissociation between the neural underpinnings of parietally distributed late cue-locked P3 and target P3 activations (Barcelo et al., 2002, 2006). These transient P3-like activations did not capture an important portion of behavioral mixing costs in the Switch task. Next we discuss these data in the light of the recent task-switching literature.

\section{Restart costs and 'early' cue-locked P3 activity}

In accord with previous task-switching studies (Altmann, 2002; Rushworth et al., 2002; Schneider and Logan, 2006), the comparison of our Switch task with 2 perceptually identical control tasks indicated that a large portion of the overall behavioral costs consisted of switch-unspecific restart costs at the beginning of each trial sequence. Behavioral restart costs were observed only in those 2 task conditions with the largest amount of task-set information (with 6:3 S-R mappings each), and affected only the $1^{\text {st }}$ target response following the appraisal of contextual signals conveying a large amount of information for response selection (see Table 1 and Figure 2). According to our estimations, the information conveyed by black symbols in the Switch and NoGo tasks exceeded the theoretical limit for holding information in human working memory (see Figure 2; cf. Miller, 1956). Tasks showing behavioral restart costs also showed enhanced cue-locked P3 activations (Figures 3 and 4A). Neither restart costs nor cue-locked P3 activations were observed in response to the least informative black symbols in the Oddball task. These effects cannot be attributed to purely sensory processes, since black symbols were embedded in identical stimulus contexts in the 3 task conditions. Instead, these results suggest that restart costs, and the accompanying cue-locked P3 activations, were a function of response uncertainty (cf. Sutton et al., 1965), as formally estimated through the mutual information between the eliciting stimulus and the available responses within each task context (Barcelo and Knight, 2007; Barcelo et al., 2006; Koechlin et al., 2003).

Behavioral task-switching costs have been attributed to switchunspecific cue-related sensory priming and task-set encoding or retrieval (Logan and Bundesen, 2003; Schneider and Logan, 2005). In line with these proposals, restart costs in the Switch and NoGo tasks, together with the accompanying early cue-locked P3 activations, were related to the initiation of trial sequences (or 'block transitions'; cf. Fox et al., 2005), rather than to any switch-specific mechanism. However, the sensory priming hypothesis cannot explain the absence of restart costs and cue-locked P3 activations in the Oddball task, with equivalent sensory priming or cue encoding demands as the other 2 tasks (Logan and Schneider, 2006). The hypothetical effects of cue-repetition benefits could be discarded after equating the proportion of cue repetitions and cue switches (Logan and Bundesen, 2003), and because our transition cues provided a control for the potential confound between task-switching and cue-switching (Forstmann et al., 2007). Even if the encoding and/or retrieval of semantic or episodic memories could be held responsible for the restart costs and brain activations in the Switch task, this would not apply to the NoGo task, where black symbols did not demand any explicit memory operations (Logan et al., 2007). Therefore, the present results argue against an account of behavioral restart costs, and the accompanying cue-locked $\mathrm{P} 3$ activations, in terms of processing (either priming, encoding, retrieval or updating) of purely perceptual representations of visual information. Instead, these results are consistent with the existence of general-purpose, lower-level preparatory task activation processes shared by the Switch and NoGo tasks (Allport and Wylie, 2000; Altmann, 2002; Jennings and van der Molen, 2005; Schneider and Logan, 2006).

The observed pattern of behavioral restart costs, and the accompanying cue-locked P3 activations, are not consistent with the updating of purely motor representations either, because black symbols were explicitly associated to a 'no response' motor program in all 3 task conditions, and they did not demand any differential processing in our NoGo and Oddball tasks. However, the differential sequencing of motor responses in the 3 tasks determined the differential informational value of black symbols, and therefore, this factor remains the main candidate for accounting for the observed behavioral and brain modulations. It could be argued that the black symbols invested a hierarchical structure to trial sequences 
in the Switch and NoGo tasks, whereby response execution to every task stimulus was unpredictably interrupted by an infrequent symbol. In contrast, the same association of black symbols with a 'no response' did not induce such a control structure in the Oddball task, where motor responses were more sparsely and irregularly distributed over time. Accordingly, the grouping of motor responses into regular sequences of target trials could be regarded as equivalent to the spatial and temporal grouping of task stimuli for inducing hierarchical control structures (Lien and Ruthruff, 2004; Schneider and Logan, 2006). Our information theoretical estimations indicated that black symbols afforded highly informative boundaries for separating sequentially ordered groupings of target responses in the Switch and NoGo - but not the Oddball - tasks. The topographical distribution of cue-locked P3 activations over frontoparietal scalp regions overlaying pre- and post-rolandic multimodal association cortices are also consistent with a sensorimotor - rather than a purely sensory or a purely motor - nature of the neural representations being updated in response to the black symbols. This hypothesis agrees with proposals that the preparatory control of action involves activation of cognitive representations rather than sensory or motor representations (Jennings and van der Molen, 2005). This interpretation also agrees with previous accounts of behavioral costs, and cue-locked P3 activations, as influenced by hierarchical structure, motor planning, and response strategies (Donchin, 1981; Lien and Ruthruff, 2004; Schneider and Logan, 2006). From this perspective, behavioral restart costs, and the accompanying cue-locked P3 activations, could be interpreted as reflecting general purpose cortical-subcortical dynamics during the intermittent reactivation of task-set information for the preparatory regulation of action (Jennings and van der Molen, 2005; Norman and Shallice, 1986; 0'Reilly and Frank, 2006).

The unpredictable onset of black symbols arguably re-activated taskset representations (Figure 1), but only when those symbols provided informative boundaries for the preparatory regulation of action. This was a general and switch-unspecific mechanism, independent of whether the ongoing task goal was to maintain or to reconfigure the currently active S-R mapping. The direct association between restart costs and early cue-locked P3 amplitudes concurs with previous accounts of transient 'nogo P3' activity in terms of inhibitory action control (Pfefferbaum et al., 1985), in anticipation of upcoming task demands (Jennings and van der Molen, 2005; Norman and Shallice, 1986). From this perspective restart costs, and the accompanying cue-locked P3, could both reflect the mobilization of neural resources in the face of larger response demands in the Switch and NoGo tasks. The hypothesis of a common pool of attentional resources would be consistent with the strong correlations between cue- and target-locked P3 activations during task preparation and execution (Kahneman, 1973). However, such a common pool of resources would also leave unexplained a large proportion of variance in the data. The rather low correlations between P3-like activity and behavior in the most difficult (Switch) and easiest (Oddball) tasks point to a complex, possibly non-linear contribution from multiple component processes to the anticipatory preparation of action (Jennings and van der Molen, 2005).

Finally, transient P3-like activations failed to capture an important portion of switch-unspecific mixing costs in the Switch task (Monsell, 2003). Residual mixing costs were observed in target trials following both switch and repeat cues in the Switch condition only, and were mostly apparent during the third target in a trial sequence, after restart and switch costs had subsided. Mixing costs could reflect passive taskset inertia (Allport and Wylie, 2000), but also maintenance of alternative S-R mappings necessary for an imminent S-R reversal (Braver et al., 2003; Meiran, 1996). Mixing costs were captured neither by cue-locked P3 activity, nor by target P3 that showed similar amplitudes to the $3^{\text {rd }}$ target in all 3 task conditions. One likely candidate for indexing mixing costs was a sustained fronto-parietal negativity observed towards the end of the cue-to-target interval in the Switch task (not shown). Whereas mean ERP amplitudes in the NoGo and Oddball tasks returned to a zero baseline from 800 to 1500 ms post-cue onset, grand ERP waveforms in the Switch task remained below $-4 \mu \mathrm{V}$ at the onset of the $1^{\text {st }}$ target in the sequence (not shown). These sustained negativities were not part of our original hypotheses and will not be further discussed here. Similar sustained negativities overlaying frontal and parietal scalp regions during the cue-to-target interval have been reported in previous task-switching studies (Barcelo et al., 2006; Kieffaber and Hetrick, 2005; Nicholson et al., 2005), and their likely association with residual mixing costs deserves further examination in future studies.

\section{Switch costs and 'late' cue-locked P3 activity}

The brain responses to ' $x$ ' switch and '+' repeat symbols in the Switch task revealed a switch-specific enhancement of the late aspect of cue-locked P3 activity, consistent with findings from other laboratories (Kieffaber and Hetrick, 2005; Nicholson et al., 2005; Rushworth et al., 2002), and in agreement with our hypothesis of an association between specific switch costs and late cue-locked P3 activations (Barcelo et al., 2002, 2006). Information theoretical estimations indicated that ' $x$ ' switch symbols in the Switch task conveyed 1 more bit of information for response selection than '+' repeat symbols, as only the former prompted for the selection of $1 \mathrm{~S}-\mathrm{R}$ mapping out of 2 possible options (Table 2). As expected, no such behavioral nor physiological modulations were observed in the NoGo and Oddball tasks, as these did not convey any extra contextual information for response selection (i.e., they did not prompt for any task-set switching operation). The present association of specific switch costs with parietally distributed late cue-locked P3 modulations also concurs with proposals about the role of parietal association cortices in the reconfiguration of S-R mappings in memory (Rushworth and Taylor, 2006).

The comparison of early and late cue-locked P3 activations in the Switch and NoGo tasks suggests that in order to reconfigure an old S-R mapping, it may be necessary to re-activate first the currently active task-set (cf. Altmann, 2002). Although somewhat counterintuitive, the re-activation of an already active task-set may reflect a default mode of cortical-subcortical dynamics during task-set shifting operations (0'Reilly, 2006; 0'Reilly and Frank, 2006; Robbins, 2007). Active maintenance of familiar and over-learned task information is thought to be partly managed subcortically, and with limited involvement from higherordered cortical control (Malmo, 1942). In turn, the updating of task-set information in response to novel and highly informative environmental events has been shown to demand prefrontal control (Barcelo and Knight, 2007; Knight, 1984). Accordingly, the default mode of cortical-subcortical dynamics when a highly informative signal interrupts us while performing a familiar, well-rehearsed and automated task may involve uploading the ongoing task-set from subcortical to cortical memory networks in preparation for any potential cortical updating or re-adjustments (0'Reilly, 2006; O'Reilly and Frank, 2006). This proposal would explain the similar modulations of early cue-locked P3 activity in our NoGo and Switch tasks (indexing reactivation of similar task-set information), whereas late P3 modulations (indexing updating of S-R mappings) were only observed in the Switch task. This account would also explain why the late aspect of cue-locked P3 activity is always subordinated to its early aspect (Barcelo et al., 2002, 2006). Finally, this hypothesis is also consistent with the stronger correlations found between early and late cue-locked P3 amplitudes, rather than between late cue-locked P3 and target P3 amplitudes (see Table 4; cf. Polich, 2007).

The observed pattern of switch-specific switch costs cannot be easily reconciled with alternative explanations in terms of stimulus priming, or as an encoding advantage of cue repetitions over cue switches (Logan and Bundesen, 2003). The hypothesis of task-set inertia would be more consistent with sustained brain activations rather than with the observed transient modulation in late - but not early - cue-locked P3 (Allport and Wylie, 2000; Altmann, 2002). Moreover, if the enhanced late cue-locked P3 indexed passive interference from the old S-R mapping, we would expect a direct association with behavioral switch costs. On the contrary, our regression analyses replicated previous findings of an 
inverse association, with larger late cue-locked P3 predicting smaller switch-specific behavioral costs (cf. Barcelo et al., 2006). The present evidence suggests that switch-specific costs, and the accompanying late cue-locked P3 activations, reflect the updating of S-R mappings from the active task set in preparation for the next trial sequence (Barcelo et al., 2002; Rushworth et al., 2002). These switch specific cue-locked P3 modulations meet the 2 criteria established by Meiran (1996) to distinguish task-preparation from task-execution processes, namely (1) late cue-locked P3 activations were specific to switch cues in the Switch task, and (2) they occurred in advance of response execution.

In accord with previous claims that switch-specific costs are 'vanishingly small' (Logan et al., 2007), we found rather moderate switch costs compared with the portion of switch-unspecific (both restart and mixing) costs in the Switch task. However, our information theoretical analyses suggested that switch cues conveyed only a limited amount of contextual information (1 bit) for updating S-R mappings in working memory, compared with the total amount of information conveyed by the same cues for response selection (Figure 2, Table 1). One corollary from these analyses is that larger amounts of contextual uncertainty for updating the active S-R mappings - relative to the total amount of task-set information - may result in larger switch-specific costs and larger late cue-locked P3 amplitudes (Barcelo et al., 2006).

Finally, we found evidence of an interaction between restart and switch costs in the Switch task, where switch-specific costs were significantly larger in the $1^{\text {st }}$ compared to the $2^{\text {nd }}$ target trials (Figure 3 ). One interpretation of this interaction is that the neural substrates of switch and restart costs draw from a common pool of subcortical resources (e.g., common neuromodulatory systems in the brainstem; Nieuwenhuis et al., 2005; Pfaff, 2006; Soltani and Knight, 2000), even though they each could be ultimately contributed by different cortical mechanisms. Another possibility is that switch costs relate to higher-level cortical S-R reconfiguration, but this depends on a general-purpose arousal mechanism for re-activating task information (Robbins, 2007). Yet another compatible possibility is that the highly informative ' $x$ ' switch cues (Figure 2) momentarily overloaded memory capacity, carrying over any cortical S-R reconfiguration to the $2^{\text {nd }}$ target trial. Whatever the cause for this interaction, the classic hypothesis of a common pool of attentional resources (Kahneman, 1973) cannot easily account for the 3 types of behavioral costs (switch, restart, mixing), and the dissociation between early and late cue-locked P3 and target P3 activations disclosed here (Jennings and van der Molen, 2005).

\section{Task-set implementation and target P3 activity}

Target-locked P3 activations suggested that behavioral task-switching costs reflect a combination of different neural mechanisms encompassing both the preparation and execution stages of task performance (Jennings and van der Molen, 2005; Rubinstein et al., 2001). The pattern of target P3 activations during task-set implementation - and their association with behavior - differed markedly from cue-locked P3 activations during the preparatory period (cf. Barcelo et al., 2002). This double dissociation was reflected in the distinct scalp distribution of peak P3-like amplitudes across the 3 task conditions (Figures 6 and 7). The analysis of sequential target effects in the Switch and NoGo tasks revealed reduced target P3 amplitudes to $1^{\text {st }}$ target trials following a black symbol, with no further changes between $2^{\text {nd }}$ and $3^{\text {rd }}$ targets trials. In accord with previous studies, these effects occurred regardless of the meaning of switch vs. repeat cues for updating the current S-R mappings (Barcelo et al., 2000, 2002; Kieffaber and Hetrick, 2005; Nicholson et al., 2005). These target P3 modulations suggested that switch-unspecific mechanisms responsible for restart costs also extended their influence to the execution stage of task performance. Thus, reduced $1^{\text {st }}$ target P3 amplitudes in the Switch and NoGo tasks seemed to reflect momentary lesser control (due to residual uncertainty) following the onset of highly informative signals that overloaded working memory capacity (Figure 2). Except for this momentary reduction in $1^{\text {st }}$ target P3 amplitudes in the 2 most difficult tasks, mean target P3 amplitudes did not differ between the 3 task conditions. In agreement with our estimations of the mutual information of targets for response selection, target-locked P3 activations also suggested that similar attentional resources were allocated to the processing of visual targets in all 3 task conditions (Figures 2 and 4).

The negative correlations found between target P3 amplitudes and all behavioral indexes (restart costs, switch costs and mean RTs), indicated that larger target P3 amplitudes indexed a more efficient and faster implementation of task-set information (the task rules). This association is consistent with previous task-switching studies where a gradual improvement in behavioral efficiency was accompanied by a gradual increase in target P3 amplitude along successive repetition trials (Barcelo et al., 2000, 2002; Kieffaber and Hetrick, 2005; Nicholson et al., 2005; Rushworth et al., 2002). These results suggest that target P3 activity reflects the exploitative implementation of task-set information, as functionally and anatomically distinct to the resolution of response uncertainty indexed by cue-locked P3 activations (Barcelo and Knight, 2007; Barcelo et al., 2006). These two functionally distinct P3-like components can be easily mistaken in selective attention and oddball tasks, where the brain activations recorded to improbable targets reflect a mixture of exploratory (uncertainty resolution) and exploitative (task execution) processes (Donchin, 1981; Karayanidis et al., 2003). Undoing this potential confound will help clarify a long-standing dispute about the functional role of brain P3-like potentials (Falkenstein et al., 1994; Verleger, 1988), and seems a prerequisite for elucidating their distinct anatomy (Barcelo and Knight, 2007; Knight, 1984), neurochemistry (Nieuwenhuis et al., 2005), and clinical applications (Polich, 2007).

\section{Mutual S-R information and the preparatory control of action}

The present results agree with the extant literature about the existence of both switch-specific and switch-unspecific processes that contribute to overall task-switching costs. Our information estimations revealed two sources of behavioral costs that corresponded with the allocation of attentional resources at two levels in a putative hierarchy of control: (1) during the intermittent re-activation of task-set information, and (2) and during the updating of specific S-R mappings in working memory. These results lend partial support to both sides of the controversy about the role of executive vs. non-executive control processes in task switching (Allport and Wylie, 2000; Logan and Bundesen, 2003; Meiran, 1996; Monsell, 2003). More importantly, this information theoretic approach has heuristic value for making predictions about the presence or absence of behavioral costs across a wide variety of task situations based on the history of contextual contingencies between response demands and environmental events. In accordance with the hypothesis of selection-for-action (Allport, 1987; Norman and Shallice, 1986), our information estimations suggest that the informational content of sensory signals depends on the history of responses associated with those signals, a notion best captured with the concept of mutual S-R information (Koechlin and Summerfield, 2007). From this perspective, it is probably inaccurate to ascribe the present task-switch costs to either purely exogenous sensory processes (Logan and Bundesen, 2003), nor to a purely endogenous act of executive control (Meiran, 1996). Instead, the present results suggest that the informational content of environmental events depends on their contextual association with previous and anticipated response demands. For the same reason, the present behavioral and physiological results do not fit well with classic dichotomous taxonomies of attended vs. ignored (Donchin, 1981), task-relevant vs. task-irrelevant (Näätänen, 1990; Posner and Petersen, 1990), or voluntary vs. involuntary processing modes (Kahneman, 1973). These dualistic classifications of task events rely on intuitive notions of information processing, and do not consider the history of contextual S-R associations which confer exogenous signals with their informational value for goal-oriented behavior. The information conveyed by the cumulative history of $\mathrm{S}-\mathrm{R}$ associations within a particular task context, as formally described through information theoretical measures 
(Koechlin and Summerfield, 2007), could result in a more flexible and accurate predictor of brain-behavior interactions.

We confirmed and extended previous ERP findings by demonstrating an association of behavioral restart and switch costs with the early and late aspects, respectively, of a cognitive 'P300' potential that has long been related to the resolution of uncertainty (Sutton et al., 1965). Sutton and collaborators (1965), and many authors thereafter (Donchin, 1981; Johnson, 1986), attributed the observed P300 modulations to the resolution of stimulus uncertainty. However, those earlier observations can be also explained in terms of the subjects' response uncertainty about incoming stimulation, based on the history of past S-R associations. The present cue-locked P3 activations, and the accompanying behavioral costs, seemed to be a function of the response uncertainty generated in 3 identical stimulus contexts calling for different response demands, as estimated through the mutual information (also transmitted information Miller, 1956; see Figure 2) between black symbols and their contextually associated response demands. In order to reach this conclusion, it was important to rely on quantitative - even if very simple - estimations of mutual S-R information. These estimations were facilitated by the use of simpler sets of stimuli and responses than in our previous task-set switching studies. In turn, it would have been questionable to describe and explain the present behavioral and ERP results based just on our intuitive notion about the "effort", "demands", "difficulty" or "saliency" of task events. All these concepts can be subsumed within formal estimations of mutual S-R information, which also offered us a deeper insight into the nature of information processing stages. For instance, our information estimations and target P3 potentials suggested that similar attentional resources were allocated to the processing of visual targets in all 3 task conditions (Figures 2 and 4). On the contrary, larger attention and "effort" were devoted to the appraisal of the highly informative black symbols (Kahneman, 1973). In spite of the intuitive association of cognitive P300 potentials with information theoretical concepts ever since their discovery (Donchin, 1981; Johnson, 1986; Sutton et al. 1965), most past ERP studies did not formally estimate the mutual information between sensory, motor, and sensorimotor representations along a putative hierarchy of cognitive control (cf. Barcelo and Knight, 2007). This new approach allowed us to disclose a critical dissociation between transient P3-like activations associated with the exploratory resolution of uncertainty during the preparatory control of action (i.e., cue-locked P3, P300, novelty P3), as distinct from the exploitative implementation of task-set information during task execution (target P3). These two functionally different P3-like components may overlap, and thus be easily mistaken, in the brain responses to improbable targets in oddball tasks, which often reflect a mixture of task preparation and task execution processes (Falkenstein et al., 1994; Nicholson et al., 2005; Polich, 2007; Verleger, 1988). Information theoretical estimations may contribute to undoing this potential confound in future ERP and brain imaging studies.

Here we utilized an information theoretical model adopted from brain imaging studies of prefrontal executive function in humans (Koechlin and Summerfield, 2007). In spite of the limited spatial resolution of scalprecorded brain potentials, the modulations of the 'frontal' aspect of cue-locked P3 activations were compatible with the role of lateral prefrontal cortex in the elicitation of cognitive P300 potentials (Barcelo and Knight, 2007; Knight, 1984). The analyses of scalp topography shown in Figure 5 suggested distinct distributions of neural sources across anterior and posterior cortical regions, supporting the functional segregation of early and late cue-locked P3, as well as of target P3 activations. However, the strong correlations found among all these P3-like subcomponents also lent support to the hypothesis that they may be modulated by common neuromodulatory systems in the brainstem (Nieuwenhuis et al., 2005; Ranganath and Rainier, 2003; Soltani and Knight, 2000), even though they each be ultimately related to different cortical mechanisms. The strongest correlations among transient P3-like activations were observed in the NoGo and Switch tasks, probably reflecting a well coordinated arousal response during the preparation and execution of the most complex tasks (Jennings and van der Molen, 2005; Pfaff, 2006). The double dissociations found in behavioral costs and the accompanying P300 modulations do not fit well with a classic model of general arousal (Kahneman, 1973), nor with a unique neuromodulatory system (Nieuwenhuis et al., 2005). Instead, these observations agree with the hypothesis that distinct lower- and higher-ordered control mechanisms depend on appropriate subcortical inputs from several neuromodulatory systems (Robbins, 2000, 2007). The functional segregation of distinct P3-like activations disclosed here may help define the contribution from different neuromodulatory systems to distinct cognitive P3-like potentials related to task-set switching and implementation. Therefore, the pattern of behavioral and brain responses disclosed here are probably best explained in terms of mutually informed cortical-subcortical dynamics during task preparation and execution (Barcelo and Knight, 2007; 0'Reilly and Frank, 2006; Robbins, 2000).

Finally, our simple information estimations did not account for several sources of variability in the data (i.e., residual mixing costs and sustained negativities towards the end of cue-to-target intervals in the Switch task). Further research will be necessary to clarify whether these sustained negativities, as well as transient target P3 activations, are also amenable to information theoretical analyses. Future studies should address whether the task preparation processes disclosed here reflect re-activation of an old task-set (Altmann, 2002), inhibition of a recently active task-set (Allport and Wylie, 2000), or competition between alternative processing pathways (Monsell, 2003). The functional and anatomical regularities of cognitive $\mathrm{P} 300$ potentials described in this and in previous task-switching studies, over and above the different sensory modalities, response demands, stimulus materials and task procedures, all lend support to the idea that cue-locked P3 potentials reflect fundamentally similar cognitive and neural mechanisms related to the resolution of response uncertainty (Barcelo and Knight, 2007; Barcelo et al., 2006), during task-set stopping and shifting (Robbins, 2007), and whose general principles could be eventually furnished into a general theory for the preparatory control of action (Jennings and van der Molen, 2005; Koechlin and Summerfield, 2007; Norman and Shallice, 1986).

\section{CONFLICT OF INTEREST STATEMENT}

The authors declare that the research was conducted in the absence of any commercial or financial relationships that could be construed as a potential conflict of interest.

\section{ACKNOWLEDGEMENT}

Funded by the Spanish Ministerio de Educación y Ciencia (SEJ2007-61728).

\section{APPENDIX}

See Tables A1-A3.

Table A1. Stimulus entropy $H(S)=-\sum_{i=1}^{i=6} p\left(s_{i}\right) \cdot \log _{2} p\left(s_{i}\right)$.

\begin{tabular}{llll}
\hline & \multicolumn{3}{c}{ All tasks } \\
\cline { 2 - 4 } Stimulus codes & $\boldsymbol{H ( s )}$ & $\boldsymbol{p}(\boldsymbol{s})$ & $\log _{\mathbf{2}} \boldsymbol{P}(\mathbf{s})$ \\
\hline$s 1$ & 0.22 & 0.05 & -4.29 \\
$s 2$ & 0.22 & 0.05 & -4.29 \\
$s 3$ & 0.48 & 0.22 & -2.16 \\
$s 4$ & 0.48 & 0.22 & -2.16 \\
$s 5$ & 0.48 & 0.22 & -2.16 \\
$s 6$ & 0.48 & 0.22 & -2.16 \\
$H(S)=$ & 2.37 & &
\end{tabular}


Table A2. Response entropy $H(R)=-\sum_{i=0}^{i=2} p\left(r_{i}\right) \cdot \log _{2} p\left(r_{i}\right)$.

\begin{tabular}{|c|c|c|c|c|c|c|}
\hline \multirow[t]{2}{*}{ Response codes } & \multicolumn{3}{|c|}{ Oddball task } & \multicolumn{3}{|c|}{ NoGo and Switch tasks } \\
\hline & $H(r)$ & $p(r)$ & $\log _{2} p(r)$ & $H(r)$ & $p(r)$ & $\log _{2} p(r)$ \\
\hline 10 & 0.47 & 0.55 & -0.86 & 0.34 & 0.10 & -3.29 \\
\hline$\Pi$ & 0.52 & 0.45 & -1.16 & 0.52 & 0.45 & -1.16 \\
\hline 12 & - & - & - & 0.52 & 0.45 & -1.16 \\
\hline$H(R)=$ & 0.99 & & & 1.37 & & \\
\hline
\end{tabular}

Table A3. Mutual information $I\left(s_{i}, r_{j}\right)=\log _{2} p\left(s_{i}, r_{j}\right)-\log _{2} p\left(s_{i}\right) \cdot p\left(r_{j}\right)$. Only the $S-R$ mappings for 'shape' classifications are illustrated here for simplicity.

\begin{tabular}{|c|c|c|c|c|c|c|}
\hline \multirow[t]{2}{*}{ Stimulus codes } & \multicolumn{3}{|c|}{ Oddball task } & \multicolumn{3}{|c|}{ NoGo and Switch tasks } \\
\hline & S-R codes & Joint $p(s, r)$ & $I(s, r)$ & $S-R$ codes & Joint $p(s, r)$ & $I(s, r)$ \\
\hline$s 1$ & $s 1-r 0$ & 0.05 & 0.86 & $s 1-r 0$ & 0.05 & 3.29 \\
\hline$s 2$ & $s 2-r 0$ & 0.05 & 0.86 & $s 2-r 0$ & 0.05 & 3.29 \\
\hline$s 3$ & $s 3-r 0$ & 0.22 & 0.86 & $s 3-r 1$ & 0.22 & 1.16 \\
\hline$s 4$ & $s 4-r 0$ & 0.22 & 0.86 & $s 4-r 1$ & 0.22 & 1.16 \\
\hline$s 5$ & $s 5-r 1$ & 0.22 & 1.16 & $s 5-r 2$ & 0.22 & 1.16 \\
\hline$s 6$ & $s 6-r 1$ & 0.22 & 1.16 & $s 6-r 2$ & 0.22 & 1.16 \\
\hline
\end{tabular}

\section{REFERENCES}

Allport, A. (1987). Selection for action: some behavioral and neurophysiological considerations of attention and action. In Perspectives on Perception and Action H. Heuer and A. F. Sanders, eds (Hillsdale, NJ, Lawrence Erlbaum Associates) pp. 395-419.

Allport, A., and Wylie, G. (2000). Task-switching, stimulus-response bindings and negative priming. In Control of Cognitive Processes: Attention and Performance XVIII, S. Monsell and J. Driver, eds (Cambridge, MA, MIT Press), pp. 35-70.

Altmann, E. M. (2002). Functional decay of memory for tasks. Psychol. Res. 66, 287-297.

Barcelo, F. (2003). The Madrid card sorting test (MCST): a task switching paradigm to study executive attention with event-related potentials. Brain Res. Brain Res. Protoc. 11, 27-37.

Barcelo, F., Escera, C., Corral, M. J., and Perianez, J. A. (2006). Task switching and novelty processing activate a common neural network for cognitive control. J. Cogn. NeurosCi. 18, 1734-1748.

Barcelo, F., and Knight, R. T. (2007). An information theoretical approach to contextual processing in the human brain: evidence from prefrontal lesions. Cereb. Cortex 17 i51-i60.

Barcelo, F., Muñoz-Céspedes, J. M., Pozo, M. A., and Rubia, F. J. (2000). Attentiona set shifting modulates the target $\mathrm{P} 3 \mathrm{~b}$ response in the Wisconsin card sorting test. Neuropsychologia 38, 1342-1355.

Barcelo, F., Periañez, J. A., and Knight, R. T. (2002). Think differently: a brain orienting response to task novelty. Neuroreport 13, 1887-1892.

Braver, T. S., Reynolds, J. R., and Donaldson, D. I. (2003). Neural mechanisms of transient and sustained cognitive control during task switching. Neuron 39, 713-726.

Donchin, E. (1981). Surprise! Surprise? Psychophysiology 18, 493-513.

Falkenstein, M., Hohnsbein, J., and Hoormann, J. (1994). Effects of choice complexity on different subcomponents of the late positive complex of the event-related potential. Electroencephalogr. Clin. Neurophysiol. 92, 148-160.

Forstmann, B. U., Brass, M., and Koch, I. (2007). Methodological and empirical issues when dissociating cue-related from task-related processes in the explicit taskcuing procedure. Psychol. Res. 71, 393-400.

Fox, M. D., Snyder, A. Z., Barch, D. M., Gusnard, D. A., and Raichle, M. E. (2005). Transient BOLD responses at block transitions. Neuroimage 28, 956-966.

Friedman, D., Cycowicz, Y. M., and Gaeta, H. (2001). The novelty P3: an event-related brain potential (ERP) sign of the brain's evaluation of novelty. Neurosci. Biobehav. Rev. 25, 355-373.

Jennings, J. R., and van der Molen, M. W. (2005). Preparation for speeded action as a psychophysiological concept. Psychol. Bull. 131, 434-459.

Johnson, R. (1986). A triarchic model of P300 amplitude. Psychophysiology 23, 367-384.

Kahneman, D. (1973). Attention and Effort. Prentice Hall, NJ, Englewood-Cliffs.

Karayanidis, F., Coltheart, M., Michie, P. T., and Murphy, K. (2003). Electrophysiological correlates of anticipatory and poststimulus components of task switching Psychophysiology 40, 329-348.
Kieffaber, P. D., and Hetrick, W. P. (2005). Event-related potential correlates of task switching and switch costs. Psychophysiology 42, 56-71.

Knight, R. T. (1984). Decreased response to novel stimuli after prefrontal lesions in man. Electroencephalogr. Clin. Neurophysiol. 59, 9-20.

Koechlin, E., Ody, C., and Kouneiher, F. (2003). The architecture of cognitive control in the human prefrontal cortex. Science 302, 1181-1185.

Koechlin, E., and Summerfield, C. (2007). An information theoretical approach to prefrontal executive function. Trends Cogn. Sci. 11, 229-235.

Konishi, S., Donaldson, D. I., and Buckner, R. L. (2001). Transient activation during block transition. Neuroimage 13, 364-374.

Kopp, B., Tabeling, S., Moschner, C., and Wessel, K. (2006). Fractionating the neural mechanisms of cognitive control. J. Cogn. Neurosci. 18, 949-965.

Lien, M. C., and Ruthruff, E. (2004). Task switching in a hierarchical task structure: evidence for the fragility of the task repetition benefit. J. Exp. Psychol. Learn. Mem. Cogn. 30, 697-713.

Logan, G. D., and Bundesen, C. (2003). Clever homunculus: is there an endogenous act of control in the explicit task-cuing procedure? J. Exp. Psychol. Hum. Percept. Perform. 29, 575-599.

Logan, G. D., and Schneider, D. W. (2006). Priming or executive control? Associative priming of cue encoding increases "switch costs" in the explicit task-cuing procedure. Mem. Cogn. 34, 1250-1259.

Logan, G. D., Schneider, D. W., and Bundesen, C. (2007). Still clever after all these years: searching for the homunculus in explicitly cued task switching. J. Exp. Psychol. Hum. Percept. Perform. 33, 978-994.

Malmo, R. B. (1942). Interference factors in delayed response in monkeys after removal of the frontal lobes. J. Neurophysiol. 5, 295-308.

Meiran, N. (1996). Reconfiguration of processing mode prior to task performance. J. Exp. Psychol. Learn. Mem. Cogn. 22, 1423-1442.

Miller, G. A. (1956). The magical number seven plus or minus two: some limits on our capacity for processing information. Psychol. Rev. 63, 81-97.

Monsell, S. (2003). Task switching. Trends Cogn. Sci. 7, 134-140.

Näätänen, R. (1990). The role of attention in auditory information processing as revealed by event-related potentials and other brain measures of cognitive function. Behav. Brain Sci. 13, 201-288.

Nicholson, R., Karayanidis, F., Poboka, D., Heathcote, A., and Michie, P. T. (2005). Electrophysiological correlates of anticipatory task-switching processes. Psychophysiology 42, 540-554.

Nieuwenhuis, S., Aston-Jones, G., and Cohen, J. D. (2005). Decision making, the P3, and the locus coeruleus-norepinephrine system. Psychol. Bull. 131, 510-532.

Norman, D. A., and Shallice, T. (1986). Attention to action: willed and automatic control of behavior. In Consciousness and Self-Regulation, R. J. Davidson, G. E. Schwartz and D. Shapiro, eds (New York, NY, Plenum Press).

0'Reilly, R. C. (2006). Biologically based computational models of high-level cognition. Science 314, 91-94. 
O'Reilly, R. C., and Frank, M. J. (2006). Making working memory work: a computationa model of learning in the prefrontal cortex and Basal Ganglia. Neural Comput. 18, 283-328.

Pfaff, D. (2006). Brain Arousal and Information Theory. Harvard, Harvard University Press.

Pfefferbaum, A., Ford, J. M., Weller, B. J., and Kopell, B. S. (1985). ERPs to response production and inhibition. Electroencephalogr. Clin. Neurophysiol. 60, 423-434.

Polich, J. (2007). Updating P300: an integrative theory of P3a and P3b. Clin. Neurophysiol. $118,2128-2148$.

Posner, M. I., and Petersen, S. E. (1990). The attention system of the human brain. Annu. Rev. Neurosci. 13, 25-42.

Ranganath, C., and Rainier, G. (2003). Neural mechanisms for detecting and remembering novel events. Nat. Rev. Neurosci. 4, 193-202.

Robbins, T. W. (2000). Chemical neuromodulation of frontal-executive functions in humans and other animals. Exp. Brain Res. 133, 130-138.

Robbins, T. W. (2007). Shifting and stopping: fronto-striatal substrates, neurochemical modulation and clinical implications. Philos. Trans. R. Soc. Lond., B, Biol. Sci. 362 , 917-932.

Rubinstein, J. S., Meyer, D. E., and Evans, J. E. (2001). Executive control of cognitive processes in task switching. J. Exp. Psychol. Hum. Percept. Perform. 27, 763-797.
Rushworth, M. F., Passingham, R. E., and Nobre, A. C. (2002). Components of switching intentional set. J. Cogn. Neurosci. 14, 1139-1150.

Rushworth, M. F., and Taylor, P. C. (2006). TMS in the parietal cortex: updating representations for attention and action. Neuropsychologia 44, 2700-2716.

Schneider, D. W., and Logan, G. D. (2005). Modeling task switching without switching tasks: a short-term priming account of explicitly cued performance. J. Exp. Psychol. Gen. 134, 343-367.

Schneider, D. W., and Logan, G. D. (2006). Hierarchical control of cognitive processes: switching tasks in sequences. J. Exp. Psychol. Gen. 135, 623-640.

Shannon, C. E. (1948). A mathematical theory of communication. Bell Syst. Tech. J. 27, 379-423, 623-656.

Soltani, M., and Knight, R. T. (2000). Neural origins of the P300. Crit. Rev. Neurobiol. 14, 199-224.

Sutton, S., Braren, M., Zubin, J., and John, E. R. (1965). Evoked-potential correlates of stimulus uncertainty. Science 150, 1187-1188.

Sutton, S., Tueting, P., Zubin, J., and John, E. R. (1967). Information delivery and the sensory evoked potential. Science 155, 1436-1439.

Verleger, R. (1988). Event-related potentials and cognition: a critique of the context updating hypothesis and an alternative interpretation of P3. Behav. Brain Sci. 11, 343-356. 